\title{
The Effects of GluA1 Deletion on the Hippocampal Population Code for Position
}

\author{
Evgeny Resnik, ${ }^{1,2 \star}$ James M. McFarland, ${ }^{3,4 \star}$ Rolf Sprengel, ${ }^{1}$ Bert Sakmann, ${ }^{2,6}$ and Mayank R. Mehta ${ }^{4,5}$ \\ Departments of ${ }^{1}$ Molecular Neurobiology and ${ }^{2}$ Cell Physiology, Max Planck Institute for Medical Research, D-69120 Heidelberg, Germany, ${ }^{3}$ Department of \\ Physics, Brown University, Providence, Rhode Island 02912, ${ }^{4}$ Department of Physics and Astronomy, Integrative Center for Learning and Memory and \\ ${ }^{5}$ Departments of Neurology and Neurobiology, University of California at Los Angeles, Los Angeles, California 90095, and ${ }^{6}$ Max Planck Institute for \\ Neurobiology, D-82152 Munich, Germany
}

The GluA1 subunit of AMPA receptors (AMPARs) is critical for hippocampal synaptic transmission and plasticity. Here, we measured the activity of single units from the CA1 region of the hippocampus while GluA1 knock-out $\left(G l u A 1^{-/}\right)$and wild-type (WT) mice traversed a linear track. Although overall firing rates were similar, $G l u A 1^{-/-}$neurons were more likely to spike in bursts, but at lower burst frequencies, compared with WT neurons. GluA1 ${ }^{-1-}$ neurons showed large reductions in all measures of spatial and directional selectivity compared with WT neurons. Consistent with these alterations of single-neuron properties, the accuracy of the population code for position was substantially reduced in $\mathrm{GluA1}^{-/-}$, yet it is predicted to approach the accuracy of WT with increasing population size. The absolute representation of space, independent of movement direction, was greatly diminished in $G l u A 1^{-/-}$mice and is predicted to remain reduced even for larger populations. Finally, we found that the rate maps of $G l u A 1^{-/-}$neurons showed increased trial-by-trial variability but reduced experiential plasticity compared with the WT. These results reveal the critical contribution of GluA1-containing AMPARs to individual place cells and the hippocampal population code for space, which could explain the selective behavioral impairments observed in these mice.

\section{Introduction}

Since the discovery by O'Keefe and Dostrovsky (1971) of hippocampal cells in the rodent that fire in a spatially selective manner ("place cells"), and the subsequent discovery of spatially selective hippocampal neurons in a number of other animals (Rolls et al., 1997; Siegel et al., 2005; Ulanovsky and Moss, 2007) and humans (Ekstrom et al., 2003), the hippocampus has been hypothesized to provide a cognitive map of an animal's environment (O'Keefe and Nadel, 1978). Consistent with this hypothesis, hippocampal lesions impair performance on several tests of spatial processing, including learning of a fixed location (spatial reference memory) (Morris et al., 1982; Reisel et al., 2002) and navigation contingent on short-term memory (spatial working memory) (Reisel et al., 2002). The specific components of hippocampal activity necessary to mediate these spatial behaviors, however, are not well understood. Previous work manipulating

\footnotetext{
Received Dec. 25, 2011; revised April 27, 2012; accepted May 9, 2012.

Author contributions: J.M.M., B.S., and M.R.M. designed research; E.R., J.M.M., and M.R.M. performed research; R.S. contributed unpublished reagents/analytic tools; E.R. and J.M.M. analyzed data; J.M.M. and M.R.M. wrote the paper.

This work was supported by Deutsche Forschungsgemeinschaft SFB636/A4 (R.S.) and National Science Foundation Career Award 0969034, National Institutes of Health/Collaborative Research in Computational Neuroscience Grant 1-R01-MH-092925-01, the Whitehall Foundation, and the W. M. Keck Foundation (M.R.M.). We thank Peter Seeburg for a careful reading of this manuscript. Related findings were presented at the Society for Neuroscience meetings in 2006, 2008, and 2009 .

*E.R. and J.M.M. contributed equally to this work.

Correspondence should be addressed to Mayank Mehta, Departments of Physics and Astronomy, Box 951547, University of California at Los Angeles, Los Angeles, CA 90095. E-mail: MayankMehta@ucla.edu.

DOI:10.1523/JNEUROSCI.6460-11.2012

Copyright $\odot 2012$ the authors $\quad 0270-6474 / 12 / 328952-17 \$ 15.00 / 0$
}

genes involved in synaptic plasticity have shown reduced spatial selectivity (McHugh et al., 1996; Rotenberg et al., 1996) and stability (Rotenberg et al., 1996) of place cells coinciding with impaired spatial memory (Bach et al., 1995; Tsien et al., 1996). However, impairments of spatial memory have also been observed after application of NMDA receptor (NMDAR) antagonist, which did not substantially alter spatial selectivity but that did eliminate experience-dependent changes (Ekstrom et al., 2001) and reduce the long-term stability of place cells (Kentros et al., 1998), suggesting that place cell plasticity and stability could be critical components for mediating spatial memory.

Previous studies present a complex picture of how various aspects of the activity of single neurons in the hippocampus contribute to different forms of spatial learning and memory. However, the hippocampus is thought to encode position and mediate navigation using a population code (Wilson and McNaughton, 1993; Gothard et al., 1996; Leutgeb et al., 2005; Wills et al., 2005). Whereas previous studies have investigated the contribution of various cellular and molecular parameters to the spatial selectivity and stability of single cells, their effect on the population code for space has received little attention (but see $\mathrm{McHugh}$ et al., 1996). To this end, we studied the hippocampal population code for space in the dorsal CA1 region of mice lacking the GluA1 AMPAR subunit. In vitro, hippocampal neurons from these GluA1 $1^{-/-}$mice show a lack of tetanically induced long-term potentiation (LTP) (Zamanillo et al., 1999; Romberg et al., 2009), as well as an impaired fast component of theta-burst plasticity (Hoffman et al., 2002; Frey et al., 2009; Romberg et al., 2009). However, more physiologically relevant forms of plasticity, such 
as the long-term component of theta-burst plasticity, as well as spike-timing-dependent plasticity (STDP), are unaltered in the hippocampus and neocortex (Hoffman et al., 2002; Frey et al., 2009; Romberg et al., 2009). Additionally, GluA1 ${ }^{-/-}$hippocampal CA1 pyramidal neurons have strongly reduced extrasynaptic AMPA currents (Andrásfalvy et al., 2003; Romberg et al., 2009) and do not show the dendritic distancedependent scaling of excitatory synaptic strengths seen in WT neurons (Andrásfalvy et al., 2003). Behaviorally, the GluA1 ${ }^{-/-}$ mice display profound impairments on several different spatial working memory tasks but perform as well as WT animals on spatial reference memory tasks (Zamanillo et al., 1999; Reisel et al., 2002; Schmitt et al., 2003). Thus, the GluA1 ${ }^{-/-}$ mice present an opportunity to study the relationship between these different forms of spatial behavior and the hippocampal population code for space, as well as the contribution of different forms of synaptic plasticity and the dendritic organization of receptors to generating this hippocampal code.

\section{Materials and Methods}

Subjects and surgery. All experiments were performed in 11 WT (C57BL/6) and nine $\mathrm{GluA1}^{-1-}$ (Zamanillo et al., 1999) age-matched male mice. Seven of the 11 WT mice used were littermate controls, whereas the remaining WT mice came from other litters. Because none of our results significantly depended on littermate status, all WT mice were pooled for analysis. Genotypes were determined and confirmed postmortem by tail-DNA PCR analysis as described by Zamanillo et al. (1999). The mice were housed individually, maintained on a $12 \mathrm{~h}$ light/ dark cycle, and had ad libitum access to food and water unless otherwise specified. All experiments were conducted and analyzed in a doubleblind manner with respect to mouse genotype.

The mice were implanted with hyperdrives above the right dorsal CA1 region of the hippocampus (anteroposterior, $2.0 \mathrm{~mm}$; lateral, $1.5 \mathrm{~mm}$ with respect to bregma). Each hyperdrive contained up to four independently movable tetrodes and a reference electrode (Wilson and McNaughton, 1993). Tetrodes were made of four $15 \mu \mathrm{m}$ nichrome wires (Kanthal), which were twisted and heat fused together. Two stainless steel screws were implanted over the opposite frontal cortex (for anchoring) and the cerebellum (for ground). Experiments were approved by District council Karlsruhe (Department 35,76247 , license number 35.9185.81/G-60/02) and were performed in accordance with the animal welfare guidelines of the Max Planck Society.

Behavioral procedures. One week after surgery, the mice were trained to run back and forth on a linear track for food reward (sweetened milk) located at the opposite ends of the track. The track $(140 \mathrm{~cm}$ length, $3 \mathrm{~cm}$ width) was made of wood and painted black. Such a narrow track ensured that the mice walked through the same region of space when moving in both directions of the journey and across trials. Goal locations were defined as the regions within $7.5 \mathrm{~cm}$ of the locations of the reward wells. Throughout the entire training and additional recording, the mice were food restricted and maintained at $\sim 85 \%$ of their postoperative ad libitum body weights.

Electrophysiology. The tetrodes were advanced gradually over the course of many days to place them in the CA1 pyramidal cell layer. The arrival of each tetrode into the hippocampus was recognized by several criteria, including the presence of $100-300 \mathrm{~Hz}$ "ripples" in the local field potential (LFP) (O’Keefe, 1976; O’Keefe and Conway, 1978; Buzsáki et al., 1992), the polarity of "sharp waves" in the LFP (Buzsáki, 1986), and the sudden appearance of multiple simultaneously recorded cells with a complex spike discharge. During recording, the mice were attached to a unitary gain head-stage preamplifier (HS-16; Neuralynx) via a cable suspended on the supporting metal string to minimize an additional load to the animal's head. Signals were filtered and differentially amplified against the reference electrode or ground by Lynx- 8 programmable amplifiers (Neuralynx). Spikes were sampled at $32 \mathrm{kHz}$ and amplified by a factor of 5,000-10,000.
The animal's position and head direction were obtained by overhead video tracking (Cohu iDome) of two light-emitting diodes (red and blue) attached to the head stage. Video recording was made with a spatial resolution of $0.25 \mathrm{~cm} /$ pixel and a sampling rate of $50 \mathrm{~Hz}$. Daily recording sessions consisted of 8-40 laps on the linear track surrounded by rest/sleep sessions ( $20 \mathrm{~min}$ ) on an elevated platform. The platform sessions were used to confirm stability of recordings made during track running. After completion of the experiments, the mice were deeply anesthetized, and electrolesion $(200 \mu \mathrm{A}$ for $4 \mathrm{~s}$ via one channel of each tetrode with respect to the ground screw) was performed to reconstruct the position of the tetrodes.

Data analysis and unit isolation. The data were analyzed offline using custom software written in MATLAB (The MathWorks). Because the data were often not distributed normally, all results were reported using medians and interquartile ranges (IQRs), with significance tests done using the Wilcoxon rank-sum test unless otherwise stated. For all the statistical tests, a $p$ value $<0.05$ was considered to be statistically significant.

Single units were manually isolated offline using graphical clustercutting software MClust-3.4 (A. David Redish, http://redishlab.neuroscience. umn.edu/MClust/MClust.html). The procedure consisted of displaying all two-dimensional projections of the multidimensional parameter space (peak, energy, valley) for all spike waveforms and applying boundaries to each apparent unit cluster. Examples of two of six possible projections of spike amplitudes on a tetrode are shown in Figure 1, $A$ and $D$, for WT and

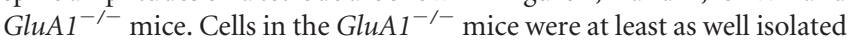
[as measured by isolation distance (Schmitzer-Torbert et al., 2005)] as those in WT (WT, median = 31, IQR = 24-39; GluA1 ${ }^{-1-}$, 41, 34-59; $\left.p=1.5 \times 10^{-8}\right)$. All detected genotype-related differences in place field characteristics were shown to be independent of unit isolation.

Putative pyramidal cells in the CA1 region were distinguished from putative interneurons using standard criteria (Ranck, 1973; Fox and Ranck, 1981; Buzsáki and Eidelberg, 1983; McNaughton et al., 1983). The classification criteria for inclusion of a unit into the pyramidal cell category was that the unit must fire at least a small number of complex spike bursts during the recording session, have spike width (peak to valley) of $\geq 250 \mu \mathrm{s}$, and an overall mean spike rate of $<2.5 \mathrm{~Hz}$ during running on the track. The subsequent analyses of spike rate maps were restricted to only those cells that had a clear refractory period in the autocorrelogram, a mean spiking rate $>0.1 \mathrm{~Hz}$, and that fired $>100$ spikes during track running. In the end, 86 of 137 WT cells and 75 of $88 \mathrm{GluA1}^{-1-}$ cells met these criteria. These cells were recorded during 52 (for WT) and 33 (for $G l u A 1^{-1-}$ ) different sessions, with up to seven (for WT) and six (for $\mathrm{GluA1}^{-/-}$) simultaneously recorded track-active cells.

Quantification of spatial spiking properties. For analysis of all spatial spiking properties, the animals' position data were first projected onto the long axis of the track. The running speed of the animal along the track was estimated by smoothing the position data using a Gaussian smoothing kernel $(\sigma=50 \mathrm{~ms})$ before computing its approximate temporal derivative. Data when the running speed of the animal was $<5 \mathrm{~cm} / \mathrm{s}$ were excluded from all analysis to ensure that "nonspatial" behaviors, such as sleeping, grooming, or rearing, did not contribute to the results. Furthermore, trials in which the mice did not run all the way from one goal location to the other were excluded from analysis.

Because most place cells on linear tracks are directional, the rightward and leftward trials were treated separately (O'Keefe and Recce, 1993; Mehta et al., 1997, 2000; Lee et al., 2004). The total number of spikes of a cell at each spatial bin $(0.5 \mathrm{~cm})$ and the time spent by the animal in that bin were computed. These spike count and occupancy maps were smoothed using a Gaussian smoothing kernel $(\sigma=2 \mathrm{~cm})$. The spike rate map was then computed by dividing the spike count in each bin by the occupancy in the corresponding bin. All spikes that occurred in the goal locations were excluded from the spike rate map calculation.

Several quantitative measures were used to describe and compare properties of the spike rate maps of the WT and GluA1 $1^{-1-}$ mice.

(1) Peak spike rate over the entire environment was defined as the maximum spike rate over all spatial bins. 
A
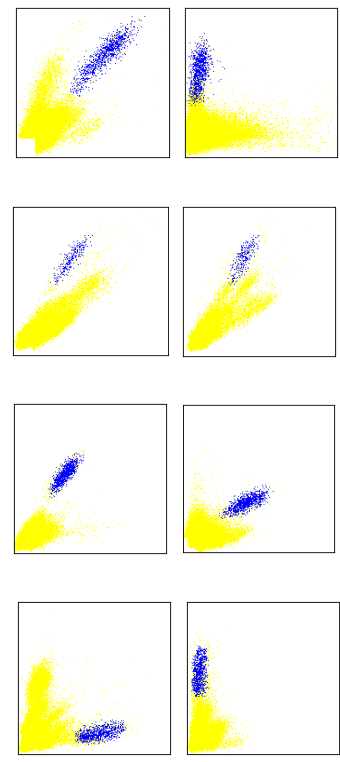

D
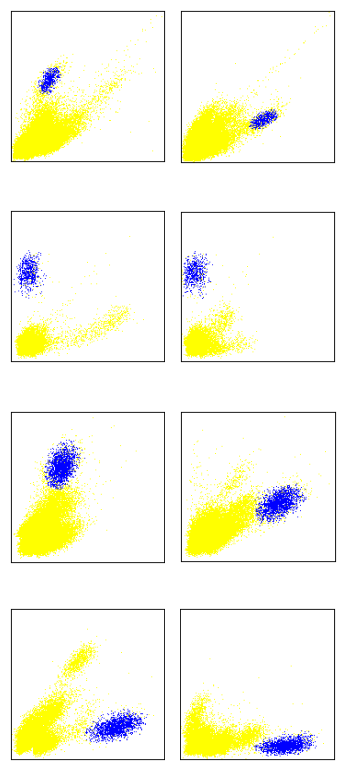

B
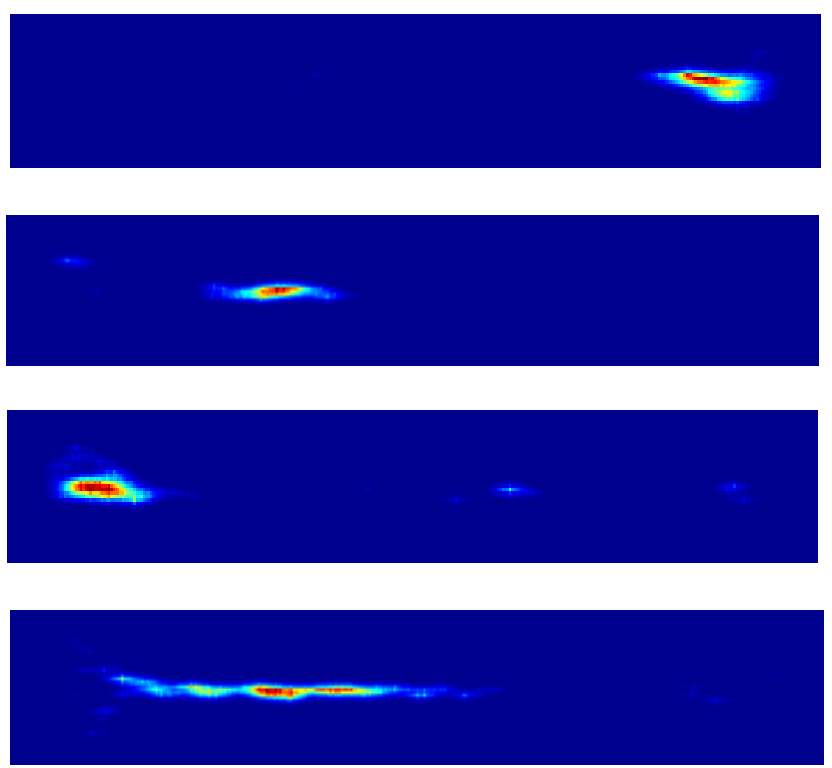

$\mathrm{E}$
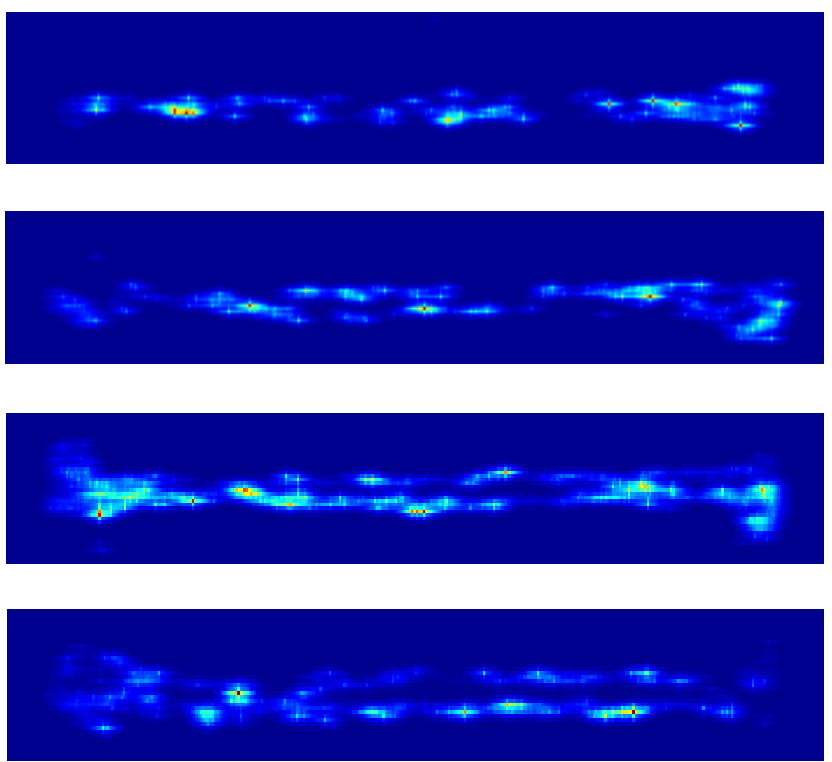

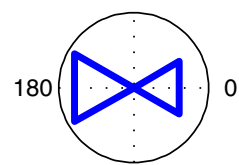

C
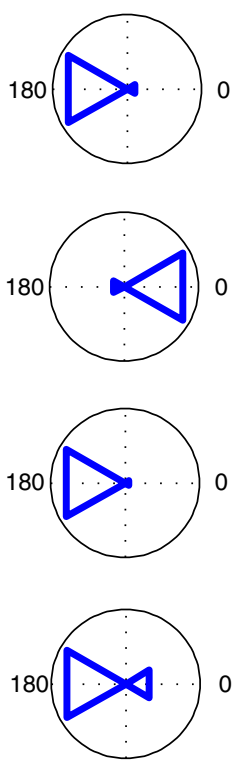

$\mathrm{F}$
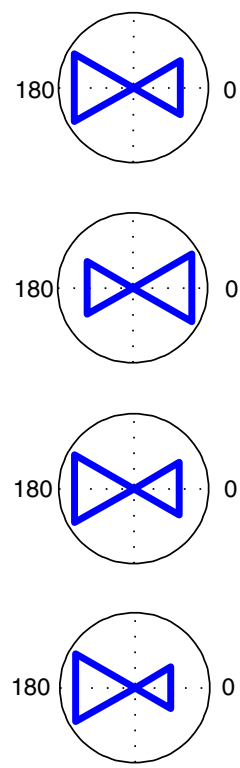

Figure 1. Comparison of unit isolation and spatial activity of four WT and $G l u A 1^{-1-}$ neurons. Data from WT are shown in $A-C$ and from $G l u A 1^{-1-}$ in D-F.A, Each yellow dot represents one spike. Each blue dot represents a spike from the depicted single unit. Two adjacent panels show the same tetrode data projected onto two different combinations of the four peak amplitudes of the tetrode. Blue dots are well isolated from the yellow dots on at least two projections, indicating good unit isolation. $\boldsymbol{B}$, Spike rate of the isolated neurons in $\boldsymbol{A}$ as a function of the mouse's position. The $x$ - and $y$-axes show the two-dimensional position of the mouse, and the color indicates the average spike rate. $C$, The spike rate of each neuron is shown as a function of the head direction of the mouse in polar coordinates in which the polar angle depicts the head direction and the radial distance depicts the spike rate of the neuron for that head direction. $\mathbf{D}-\boldsymbol{F}$, Same as $\boldsymbol{A - C}$ for $G l u A 1^{-\prime-}$ mice.

(2) Spatial information content per spike was defined by the following formula (Markus et al., 1994):

$$
I=\frac{1}{\bar{\lambda}} \sum_{i} P_{i} \lambda_{i} \log _{2}\left(\frac{\lambda_{i}}{\bar{\lambda}}\right)
$$

where $\lambda_{i}$ is the spike rate of a cell in position bin $i, P_{i}$ is the probability of occupancy in spatial bin $i$, and $\bar{\lambda}$ is the overall average spike rate (during trial running). We also estimated the "spike-position mutual information," as shown by Olypher et al. (2003). Specifically, this was defined as follows:

$$
I(X, K)=\sum_{i} \sum_{k} P_{k, x_{i}} \log \left(\frac{P_{x_{i}, k}}{P_{x_{i}} P_{k}}\right),
$$

where $X$ is the sequence of mouse positions and $K$ is the corresponding sequence of binned spike counts, $P_{x_{i}, k}$ is the joint probability of observing $k$ spikes and the mouse being in position bin $i, P_{x_{i}}$ is the marginal probability of the mouse occupying position bin $i$, and $P_{k}$ is the marginal probability of observing $k$ spikes in a time bin. For this calculation, we used time bins of $100 \mathrm{~ms}$ and spatial bins of $5 \mathrm{~cm}$. To avoid undetermined quantities appearing in the sum in Equation 2 as a result of probability 
estimates equaling 0 , we used the "K-T estimate" for the probabilities (Olypher et al., 2003) defined by the following:

$$
\hat{P}_{i}^{K T}=\frac{n_{i}+0.5}{N+0.5 J}
$$

where $n_{i}$ is the number of times outcome $i$ is observed, $N$ is the total number of observations, and $J$ is the number of unique outcomes.

(3) Direction selectivity $d$ was defined as follows:

$$
d=\left|\frac{\bar{\lambda}_{L}-\bar{\lambda}_{R}}{\bar{\lambda}_{L}+\bar{\lambda}_{R}}\right|,
$$

where $\bar{\lambda}_{L}$ is the average value of the spike rate map for leftward movements, and $\bar{\lambda}_{R}$ is the average for rightward movements. The direction selectivity defined in this way is bounded between 0 and 1 and measures the difference between the average spike rates as the mouse moved in the two directions along the track, with 1 being a maximal difference and 0 being no difference.

(4) Coherence was used to estimate the local smoothness of the spike rate maps. It was defined analogously to the definition used by Muller and Kubie (1989). First, we computed the spike count and occupancy maps using coarser 2.5-cm-wide spatial bins without any spatial smoothing. A separate, "smoothed" version of the same spike rate map was then computed by averaging with a uniform four-point (nearest + nextnearest neighbor) kernel. The coherence was then defined as the $z$-transform of the correlation coefficient between the smoothed and unsmoothed rate maps.

(5) Place fields were defined as regions in which the mean spike rate crossed above a certain threshold for $\geq 10$ contiguous bins $(5 \mathrm{~cm})$. Two different threshold values were used: either a fixed $1 \mathrm{~Hz}$ threshold (Muller et al., 1987; McHugh et al., 1996) or an adaptive threshold of $20 \%$ of the overall peak spike rate (Hollup et al., 2001; Kjelstrup et al., 2008). Two place fields were considered distinct if the average spike rate crossed below the threshold for $\geq 10$ bins $(5 \mathrm{~cm})$ between the field peaks. Place field width was defined as the distance between the outermost two bins in which the spike rate profile crossed the threshold.

(6) The signal-to-noise ratio (SNR) of a place cell was defined as:

$$
S N R=\frac{\bar{\lambda}_{s}-\bar{\lambda}_{n}}{\bar{\lambda}_{s}+\bar{\lambda}_{n}},
$$

where $\bar{\lambda}_{s}$ is the mean spike rate of the cell within all its place fields (as defined above), the so-called "signal, " and $\bar{\lambda}_{n}$ is the mean spike rate outside all the place fields, the so-called "noise." The SNR is a dimensionless number bounded between -1 and +1 , with higher values indicating more spatially restricted spiking. The SNR was calculated using place fields detected by both the $1 \mathrm{~Hz}$ and the $20 \%$ thresholds, giving qualitatively similar results. When using the $1 \mathrm{~Hz}$ threshold, one of the GluA1 ${ }^{-1-}$ cells had no place fields on the track.

(7) The rate map sparsity $\psi$ of a cell was defined as follows:

$$
\psi=1-\frac{1}{2 L} \frac{\left(\sum_{i} \lambda_{i}\right)^{2}}{\sum_{i} \lambda_{i}^{2}},
$$

where $\lambda_{i}$ is the average spike rate of the cell in position bin $i$, and $L$ is the number of spatial bins for each direction of the journey. Sparsity defined in this way is a positive, dimensionless number with a minimum value of 0 corresponding to a uniform rate map, and a maximal value of 1 $-\frac{1}{2 L}$ corresponding to a delta function rate map.

Resampling under the null hypothesis of zero spatial informationcontent. To estimate the distributions of various quantities under the null hypothesis that the spiking of the neurons contained no information about the animals' position, we used a resampling procedure similar to that used by Markus et al. (1994). For each cell, the discontinuous position and spike count time series during track-running epochs were first concatenated into vectors containing the data used for analysis (during included trials, with running speed $>5 \mathrm{~cm} / \mathrm{s}$ ). The spike count vector was then randomly shifted relative to the position vector, imposing circular boundary conditions. Because the periodic nature of the animals' track-running behavior across trials could still generate significant spatial information after such shifts, we additionally performed random circular shifts within each individual trial to randomize the relationship between position and spiking data both within and across trials. We then computed spike rate maps and the associated spatial selectivity measures from these randomized maps to generate null distributions. Importantly, these randomized rate maps preserved the temporal statistics of the spiking of each neuron and of the animals' behavior while removing any relationship between the spiking and position data. We used 500 repetitions of this resampling procedure to generate various null distributions.

Population vector analysis. Population vector analysis based on the properties of randomly sampled individual neurons is expected to be representative of the behavior of the entire neural ensemble when the number of neurons used is large. Hence, unless otherwise stated, we pooled neurons across different recording sessions and animals for population analysis, leveraging the highly systematic behavior of the animals on the linear track to analyze the population code of larger groups of neurons than were recorded simultaneously. The population vector representing the $i$ th position bin was defined as follows:

$$
\rho_{i}^{c}=\frac{\lambda_{i}^{c}}{\bar{\lambda}_{i}}
$$

where the index $c$ runs over all cells, and $\lambda_{i}^{c}$ is the value of the rate map for the $c$ th cell at position bin $i$. Normalization by the mean spike rate $\bar{\lambda}_{i}$ of each neuron prevented the population vector from being dominated by the cells with the highest spike rates. This normalization is slightly different from that used previously (Battaglia et al., 2004), although we found similar results using peak-rate normalization as well. Given this definition of the population vector at position bin $i$, the population vector sparsity $\Pi_{i}$ in spatial bin $i$ was defined as follows:

$$
\Pi_{i}=1-\frac{1}{N} \frac{\left(\sum_{c=1}^{N} \rho_{i}^{c}\right)^{2}}{\sum_{c=1}^{N}\left(\rho_{i}^{c}\right)^{2}} .
$$

Analogously to the rate map sparsity defined in Equation 6, the population vector sparsity has a minimal value of 0 , corresponding to uniform spiking across all neurons, and a maximal value of $1-\frac{1}{N}$ corresponding to spiking isolated to only one neuron.

The autocorrelation matrix of the population vectors was given by computing the Pearson's correlation coefficient between the population vectors $\bar{\rho}_{i}$ and $\bar{\rho}_{j}$ representing each pair of positions $i$ and $j$, including pairs of positions encoding opposite movement directions. As depicted in Figure 4, $A$ and $B$, the lower left and upper right quadrants of the autocorrelation matrices correspond to correlations between population vectors encoding the same movement directions, whereas the offdiagonal quadrants represent correlations between population vectors encoding opposite movement directions. The average population vector correlation as a function of the distance between the encoded positions (Fig. 4C,D) was computed by averaging the correlations across all pairs of population vectors whose encoded positions were separated by each distance. This calculation was performed separately for population vectors encoding the same (Fig. 4C) and opposite (Fig. 4D) movement directions. The standard error of the mean (SEM) was computed across all pairs of positions with a given separation distance. Although the population sizes for WT and GluA1 ${ }^{-1-}$ population vectors were different, the results of these calculations were qualitatively very similar when the WT population was randomly subsampled to be of the same size as the GluA1 ${ }^{-1-}$ population. Moreover, for the analysis of population vector correlations, the rate map for each neuron was computed without any spatial smoothing (and using coarser $2.5 \mathrm{~cm}$ bins) to avoid introducing any additional structure to the autocorrelation matrix. This was particularly important 
for computing the population vector "decorrelation distance," defined as the half-width of the central autocorrelation peak at $50 \%$ of its maximal value (correlation of 0.5 ). Other features of the population vector autocorrelation matrices were similar when using the smoothed rate maps.

Population decoding analysis. To perform population decoding of position, we used a "leave-one-out" cross-validation procedure (Rutishauser et al., 2006) to generate estimates of position from the singletrial spike rate maps of populations of neurons. Single-trial rate maps were calculated in the same way as the overall rate maps but using $2.5 \mathrm{~cm}$ spatial bins and a Gaussian smoothing $\sigma$ of $2.5 \mathrm{~cm}$. In each iteration, a random subset of neurons was first selected from the full populations of each genotype, ensuring that equal-sized populations of WT and GluA $1^{-1-}$ neurons were always compared. For each selected neuron, one trial was randomly set aside as "test data," and the remaining trials were used to train the decoding model.

Two different decoding approaches were used. In the first, the template-matching method (Wilson and McNaughton, 1993), the training data was used to compute "expected" population vectors at each spatial bin. The test data were then used to generate the "observed" population vectors. For each spatial bin, the decoded position was chosen by finding the expected population vector that maximized the correlation coefficient with the observed population vector at that position. This method thus selected a position based on the relative pattern of activity across the population of neurons. For decoding analysis, we did not use mean rate normalized population vectors. We found qualitatively similar results when using the normalized population vectors; however, decoding performance was slightly better without it.

The second decoding method was based on an explicit probabilistic model (Foldiak, 1992; Zhang et al., 1998; Pouget et al., 2000). In this case, information about the probability of observing each possible spike rate from each neuron at every position was used rather than simply matching the observed pattern of spike rates to the best-matching pattern of across-trial average rates. Specifically, for each neuron, we fit a model of the distribution of single-trial spike rates at each spatial bin based on the training data. We used a gamma distribution model for the spike rate $r$, given by the following:

$$
p(r ; \alpha, \beta)=\frac{r^{\alpha-1} e^{-\beta r} \beta^{\alpha}}{\Gamma(\alpha)},
$$

where $\alpha$ and $\beta$ are the shape and rate parameters, respectively, and $\Gamma(x)$ is the gamma function. Because the distributions of spike rates across trials tended to have an excess of zeros relative to what would be predicted from a single distribution model, we used a zero-inflated gamma model (Aitchison, 1955) given by the following:

$$
p(r ; \alpha, \beta, \delta)=\left\{\begin{array}{ll}
(1-\delta) \frac{r^{\alpha-1} e^{-\beta r} \beta^{\alpha}}{\Gamma(\alpha)} & \text { if } r>0 \\
\delta & \text { if } r=0
\end{array} .\right.
$$

This two-component mixture model separately models the probability of the spike rate being zero and the probability that the rate will take each positive value, by a mixture of a gamma distribution and a point mass at $r=0$. Thus, for a given neuron, a set of parameters, $\alpha, \beta$, and $\delta$, was fit to the distribution of single-trial spike rates at each position bin. Importantly, this approach does not assume a particular functional form for the dependence of spike rate on position but rather accommodates arbitrary spike rate maps. Because the number of model parameters is relatively large, Bayesian point estimates were used to minimize over-fitting. For $\delta$, we assumed a uniform prior and took the Bayesian posterior mean given by the following:

$$
\hat{\delta}=\frac{1+n_{0}}{2+N}
$$

where $n_{0}$ is the number of training trials in which the spike rate at the given position bin was zero, and $N$ is the total number of training trials. For the rate and shape parameters $\beta$ and $\alpha$, we assumed independent gamma priors: $\operatorname{Gamma}(a, b)$ and $\operatorname{Gamma}(c, d)$, respectively, with hyperparameters $a=1.5, b=2.0, c=1.5, d=0.2$ set by inspection of the overall distribution of single-trial spike rates. Maximum a posteriori estimates of $\alpha$ and $\beta$ were computed numerically given the following:

$$
p(\alpha, \beta \mid \text { data }) \propto \frac{1}{(\Gamma(\alpha))^{n}} \beta^{a+n \alpha-1} e^{-\beta\left(b+T_{1}\right)-d \alpha} T_{2}^{\alpha-1} \alpha^{c-1}
$$

where $T_{1}=\sum_{t} r_{t}$ and $T_{2}=\prod_{t} r_{t}$, with the sum and product running over all training trials with non-zero spike rates. Assuming independent spike rates across the neural population, the probability of observing a set of spike rates $\mathbf{r}$ given the animal is at position $x_{i}$ is then given by the following:

$$
p\left(\mathbf{r} \mid x_{i}\right)=\prod_{c} p\left(r^{c} \mid x_{i}\right)
$$

where $r^{c}$ is the spike rate of the $c$ th neuron. The decoded position for the probabilistic approach was determined by finding the $x_{i}$ which maximized $p\left(\mathbf{r} \mid x_{i}\right)$ across all position bins. We also implemented a Bayesian approach that incorporated a prior distribution on $x$ based on the animals' spatial occupancy in the training data, but the results were not significantly altered.

We used two measures to quantify decoding performance: the fraction of decoded positions that were of the incorrect movement direction and the average distance between the true and decoded positions (decoding error), given the movement direction was correctly decoded. To determine the accuracy of the absolute representation of position (independent of movement direction), we also computed the distribution of decoding errors for the incorrect movement direction by forcing the algorithm to choose among the positions for the opposite direction of the journey. Decoding errors were then defined with respect to the direction of movement (so that positive errors corresponded to predicted positions ahead of the animals' current position).

To determine the dependence of decoding accuracy on population size, we used randomly selected subpopulations of various sizes. For each population size, we estimated the decoding error for the "test trial" at each position and averaged over 50 repetitions. We then fit a power law relationship between decoding accuracy and population size of the following form:

$$
y=a N^{k}
$$

where $N$ is the population size, $y$ is the measure of decoding accuracy, and $a$ and $k$ are fit parameters.

Although previous hippocampal population decoding analyses have been based on simultaneously recorded populations of neurons, using observed spike counts across the population to decode position (Wilson and McNaughton, 1993; Zhang et al., 1998; Jezek et al., 2011), here we decoded position based on single-trial estimates of the firing rate of each neuron as a function of position, using the systematic behavior of the animals on the linear tracks to pool neurons across recording sessions. Such pooled population analysis is common and has the advantage that the population size is much larger and hence more representative of the behavior of the entire population (Georgopoulos et al., 1986; Britten et al., 1992; Stanley et al., 1999; Chechik et al., 2002). Although such pooling implicitly assumes neurons code for position independently, previous studies using simultaneously recorded neurons have made this assumption implicitly or explicitly in their decoding procedures (Zhang et al., 1998). To determine whether our population decoding analysis was likely to be strongly influenced by the pooling procedure, we also performed a decoding analysis using the largest simultaneously recorded populations of WT (seven cells) and GluA1 ${ }^{-/-}$(six cells) for comparison. For the simultaneously recorded populations, we again computed spike rate maps for each neuron using all but one randomly selected trial in each iteration. Vectors of spike counts were computed for each neuron in the left-out trial using time bins of duration $\tau$. During a given time bin, we then computed the maximum likelihood estimate of the animal's position, assuming that the spikes of each neuron were generated independently from an inhomogeneous Poisson process with conditional mean spike rates determined from the estimated rate maps (Zhang et al., 1998). Average decoding error was computed across a random sample of 
Table 1. Summary of changes in the spatial spiking properties of $\mathrm{GluA1}^{-/-}$neurons compared with WT

\begin{tabular}{|c|c|c|c|c|}
\hline & WT & GluA1 $^{-1-}$ & $\%$ Change & $p$ value \\
\hline \multicolumn{5}{|l|}{ Spatial spiking properties } \\
\hline Rate map average $(\mathrm{Hz})$ & $1.3(0.77-2.3)$ & $0.90(0.60-1.5)$ & -31 & $8.9 \times 10^{-3}$ \\
\hline Rate map peak (Hz) & $15(8.2-20)$ & $4.7(3.0-8.8)$ & -69 & $2.3 \times 10^{-13}$ \\
\hline Direction selectivity & $0.62(0.34-0.82)$ & $0.26(0.13-0.69)$ & -58 & $1.1 \times 10^{-3}$ \\
\hline Information content (bits/spike) & $1.9(1.3-2.4)$ & $0.65(0.37-1.5)$ & -66 & $7.4 \times 10^{-12}$ \\
\hline Spike-position info (bits) & $0.13(0.097-0.17)$ & $0.074(0.051-0.10)$ & -44 & $3.0 \times 10^{-12}$ \\
\hline Rate map coherence & $1.5(1.1-1.8)$ & $0.65(0.35-1.2)$ & -57 & $2.3 \times 10^{-13}$ \\
\hline Rate map sparsity & $0.8(0.70-0.86)$ & $0.48(0.33-0.75)$ & -40 & $1.6 \times 10^{-11}$ \\
\hline No. place fields/cell (1 Hz) & $2.5(2-4)$ & $4(2-5)$ & +60 & $1.9 \times 10^{-3}$ \\
\hline No. place fields/cell (20\%) & $2(1-3)$ & $5(2.25-6)$ & +150 & $2.9 \times 10^{-12}$ \\
\hline Place field size $(\mathrm{cm})(1 \mathrm{~Hz})$ & $19(9.0-32)$ & $16(7.5-29)$ & -16 & 0.082 \\
\hline Place field size $(\mathrm{cm})(20 \%)$ & $19(11-29)$ & $17(7.5-30)$ & -11 & 0.15 \\
\hline SNR (1 Hz threshold) & $0.93(0.87-0.97)$ & $0.65(0.52-0.84)$ & -30 & $2.3 \times 10^{-16}$ \\
\hline SNR (20\% threshold) & $0.87(0.80-0.93)$ & $0.68(0.60-0.82)$ & -22 & $4.8 \times 10^{-13}$ \\
\hline \multicolumn{5}{|l|}{ Rate map dynamics } \\
\hline Systematic variation (\%) & $15(11-26)$ & $5.5(4.2-10)$ & -63 & $9.6 \times 10^{-14}$ \\
\hline Stability index & $0.73(0.55-0.82)$ & $0.25(0.11-0.58)$ & NA & $3.4 \times 10^{-15}$ \\
\hline Rate distribution (r) & $0.44(0.047-0.82)$ & $0.19(-0.11-0.44)$ & NA & $5.3 \times 10^{-3}$ \\
\hline Mean rate change (\%) & $24(11-41)$ & $7.0(2.6-18)$ & -71 & $3.5 \times 10^{-6}$ \\
\hline Peak rate change (\%) & $18(5.9-38)$ & $5.1(0.9-13)$ & -72 & $1.8 \times 10^{-6}$ \\
\hline COM change $(\%)$ & $13(2.4-30)$ & $3.9(1.1-12)$ & -70 & $1.4 \times 10^{-3}$ \\
\hline Sparsity change (\%) & $20(5.2-37)$ & $4.6(1.2-19)$ & -77 & $2.4 \times 10^{-4}$ \\
\hline
\end{tabular}

Statistics are reported for spatial spiking and rate map plasticity measures as median (IQR). The percentage change is defined as (GluA1 ${ }^{-1-}-$ WT)/WT, and $p$ values are given for Wilcoxon rank-sum tests.

time bins during each trial, and distributions of average decoding errors were computed across trials. We tried a range of values for $\tau$ from $100 \mathrm{~ms}$ to $1.5 \mathrm{~s}$ and selected the value producing the smallest average decoding error for each genotype ( $900 \mathrm{~ms}$ for WT and $700 \mathrm{~ms}$ for $\mathrm{GluA1}^{-1-}$ ). Average decoding errors were relatively consistent over a large range of time bin durations.

Quantification of interspike interval distributions. The empirical interspike interval (ISI) distribution was computed for ISIs $<1 \mathrm{~s}$ using 50 logarithmically spaced bins ranging from $1 \mathrm{~ms}$ to $1 \mathrm{~s}$. The average probability of an ISI being in the range defined by each bin was then computed separately for WT and $G l u A 1^{-1-}$ cells. The depth of modulation of the difference between these average WT and $G l u A 1^{-1-}$ ISI probabilities was computed as $\left(P_{\mathrm{WT}}(\mathrm{ISI})-P_{\text {GluA } 1-/-}(\mathrm{ISI})\right) /\left(P_{\mathrm{WT}}(\mathrm{ISI})+P_{\text {GluA } 1-/-}(\mathrm{ISI})\right)$ and was smoothed slightly using a Gaussian kernel. The three ISI values in which this smoothed depth of modulation function crossed 0 were calculated and were used to define four non-overlapping ISI intervals in the range from $1 \mathrm{~ms}$ to $1 \mathrm{~s}$. The fraction of ISIs occurring within each of these ISI ranges was then computed for each cell. Only spikes fired on the track when the animal was running with sufficient speed (during the same epochs used for analysis of spatial spiking properties) were used for analysis of temporal spiking properties. To determine the relationship between ISI probabilities and spatial information, the Pearson's correlation coefficient between spatial information and ISI probability were computed using coarser ISI bins (20 logarithmically spaced bins over the same range).

Rate map plasticity and stability. Rate map plasticity and stability analyses were performed using spike rate maps that were computed for each individual trial using a slightly larger Gaussian smoothing $\sigma$ of $3 \mathrm{~cm}$. Several different measures were used to quantify the amount of acrosstrial stability and systematic change, or plasticity, in the rate maps. First, we performed a linear regression of the spike rate at each position as a function of trial number. Systematic variation was then defined as the percentage of the overall across-trial variance of the trial-by-trial rate map matrices accounted for by this set of linear models. We also computed the amount of systematic change with trial number in several different statistics of the single-trial rate maps. These included the mean spike rate, peak spike rate, center of mass (COM), and sparsity. The COM was defined as a distance relative to the departed goal location on each trial (so that changes in the COM were consistently with respect to the direction of movement in the given trial). The amount of systematic change in each of these statistics was then given by the $r^{2}$ value of a linear regression on the trial number (numbers are reported as $100 \times r^{2}$ to convey the percentage of variance explained).

To measure changes in the spatial distribution of the spike rate maps across trials, we also used a correlation-based measure of systematic change. For this, we averaged together the rate maps from the final five trials from each neuron to create a "lookup" rate map. We then computed the similarity (correlation coefficient) of each of the preceding single-trial rate maps to the lookup rate map. The correlation coefficient between trial number and this lookup correlation was taken as a measure of systematic change in the spatial distribution of the rate map of each neuron. Similarly, we computed a measure of systematic change in the population vectors by setting aside trials $11-15$ from each neuron to compute lookup population vectors. We then computed the average population vector correlation (averaged across positions) for each of the first 10 trials.

To quantify the degree of stability in the single-trial rate maps, we defined a "stability index" as follows:

$$
\frac{1}{T} \sum_{t=1}^{T} \tanh ^{-1}\left(\rho\left(\lambda_{t}, \bar{\lambda}_{-t}\right)\right)
$$

where $\rho(\cdot)$ indicates the Pearson's correlation coefficient, $\lambda_{t}$ is the rate map for trial $t, \bar{\lambda}_{-t}$ is the average rate map over all trials excluding trial $t$, and $T$ is the total number of trials.

To determine whether the rate map of a given neuron exhibited a significant amount of systematic change, we randomly shuffled the trials and recomputed each measure of systematic rate map changes. Five hundred repetitions of this trial-shuffling procedure were used to generate null distributions of each statistic.

Analysis of covariance. The aoctool function of MATLAB was used to perform analysis of covariance (ANCOVA). In all cases, we verified that there were no significant interactions between group and covariate effects, and fit models without interaction terms. To determine whether the mean rate distribution change (correlation coefficient between single-trial lookup correlation and trial number) for each genotype was significantly greater than zero after accounting for the correlation between rate distribution change and the amount of systematic change in running speed, we tested whether the mean rate distribution change evaluated at zero speed change was significantly greater than zero. 


\section{Results}

Reduced spatial selectivity of GluA1 ${ }^{-/-}$neurons

Place cell activity was recorded from 11 WT $(\mathrm{C} 57 \mathrm{BL} / 6)$ and nine GluA1 ${ }^{-1-}$ mice as they ran back and forth on a linear track for food rewards at the ends. In total, 137 and 88 putative CA1 pyramidal cells were recorded from the WT and GluA1 ${ }^{-/-}$ mice, respectively. Of these, $86 \mathrm{WT}$ and 75 GluA1 $1^{-/-}$cells had sufficient spiking activity during running on the track, away from the goal locations, and all analysis was restricted to these cells (see Materials and Methods). Although most WT cells had clear, focused place fields in a small region of space, the GluA1 ${ }^{-/-}$neurons did not show clear spatial selectivity (Fig. 1). Most place fields in WT were directional, spiking primarily in one direction of the journey, whereas the GluA1 ${ }^{-/-}$cells showed poor direction selectivity. These impressions were quantitatively confirmed by computing several measures of spatial and directional selectivity (see Materials and Methods).

The direction selectivity of the hippocampal neurons showed a 2.3-fold reduction in the GluA1 $1^{-/-}$mice (Table 1). The rate code for neurons in the WT mice was also more robust than that in the GluA $1^{-/-}$mice, as evidenced by a 3.1-fold reduction in the peak spiking rate of GluA1 $1^{-/-}$neurons compared with WT (Fig. 2A). The GluA1 $1^{-1-}$ neurons showed much less spatially localized spiking compared with WT neurons, resulting in a 1.7 -fold reduction in the rate map sparsity (Fig. $2 B$ ). Furthermore, the spatial information content per spike (Markus et al., 1994; Battaglia et al., 2004) was 3.0-fold lower for GluA1 $1^{-1-}$ than WT neurons (Fig. 2C). The spike-position mutual information (Olypher et al., 2003) also showed a 1.8 -fold decrease in the GluA1${ }^{-/-}$neurons (Table 1). Moreover, the spike rate maps of $G l u A 1^{-1-}$ cells were less locally smooth, as measured by a 2.3 -fold decrease in their rate map coherence (Fig. 2D).

To test whether the spatial modulation of a neuron was greater than expected by chance, we used a resampling procedure. By randomly shifting the time series of spike counts relative to the time series of mouse positions, both within and across trials, we generated distributions of each spatial selectivity measure under the null hypothesis of no spatial modulation (see Materials and Methods). Importantly, this resampling procedure preserved the temporal structure of the spiking of the neurons and the animals' behavior while rendering the spike and position time series independent. The resampling procedure revealed that, although the spatial selectivity of $G l u A 1^{-/-}$neurons was greatly reduced relative to WT, most GluA1 $1^{-/-}$neurons still retained some significant spatial modulation. Sixty-one of $75 \mathrm{GluA1}^{-1-}$ neurons showed significant spatial information content at the $p=0.05$ level (Table 2), which was significantly less than for WT ( 85 of 86;
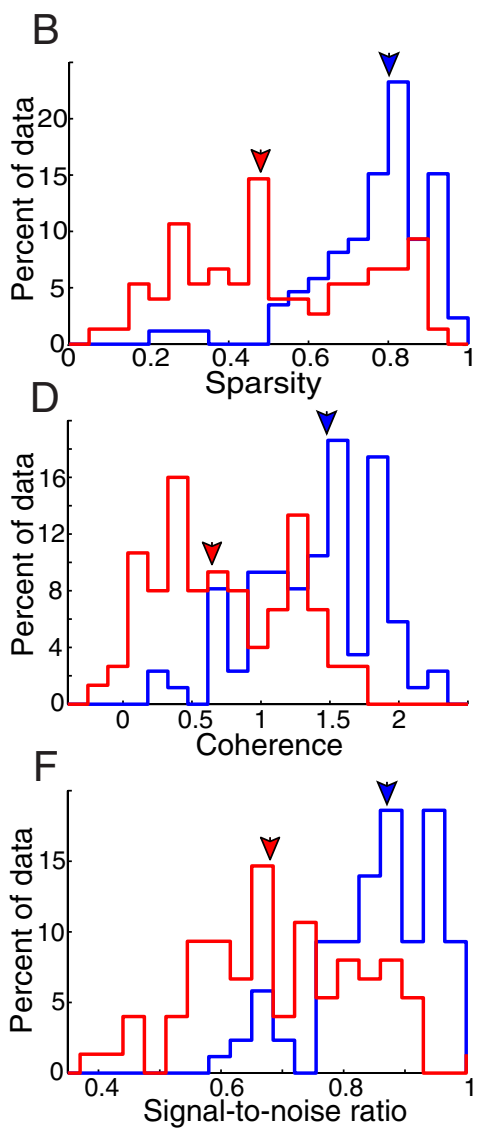

Figure 2. Impaired spatial modulation of $\mathrm{GluA} 1^{-1-}$ neurons. $\boldsymbol{A}$, Distributions of the peak spike rate for WT (blue line; $n=86$ neurons from 52 mice) and $G l u A 1^{-1-}$ (red line; $n=75$ neurons from 33 mice) cells. WT cells had significantly higher peak spike GA $1^{-1}$ - cells are indicated by the blue and red arrows, respectively. $\boldsymbol{B}$, Distributions of the rate map sparsity for WT and neurons. Rate map sparsity was significantly lower in GluAT $\quad(0.48,0.33-0.75)$ than in WT $(0.80,0.70-0.86)(p=$ cells also had smoother spike rate maps than $\mathrm{GluA1}^{-1-}$ cells, as quantified by the coherence (WT, 1.5, 1.1-1.8; GluA1 ${ }^{-1-}, 0.65$, $\left.0.35-1.2 ; p=2.3 \times 10^{-13}\right)$. $\boldsymbol{E}$, There were more than twice as many place fields per neuron in $\mathrm{GluA}^{-1-}$ than WT mice using a spike rate threshold of $20 \%$ of the peak rate of the cell (WT, 2,1-3; GluA1 ${ }^{-1-}, 5,2.3-6 ; p=2.9 \times 10^{-12}$ ). When a $1 \mathrm{~Hz}$ threshold was used, the results were similar (WT, 2.5, 2-4; GluA1 ${ }^{-1-}, 4,2-5 ; p=1.9 \times 10^{-3}$ ). $\boldsymbol{F}$, The SNR (using the $20 \%$ threshold) was higher in WT cells $(0.87,0.80-0.93)$ than in GluA1 ${ }^{-/-}$cells $(0.68,0.60-0.82)\left(p=4.8 \times 10^{-13}\right)$.

Table 2. Number of cells with significant spatial modulation and plasticity

\begin{tabular}{llc}
\hline & WT (of 86) & GluA1 \\
\hline Rate map peak (Hz) & $83(97 \%)$ & $41(55 \%)$ \\
Direction selectivity & $60(70 \%)$ & $40(53 \%)$ \\
Information content (bits/spike) & $85(99 \%)$ & $61(81 \%)$ \\
Rate map coherence & $84(98 \%)$ & $49(65 \%)$ \\
Rate map sparsity & $85(99 \%)$ & $58(77 \%)$ \\
Systematic variation & $55(64 \%)$ & $13(17 \%)$ \\
Rate distribution change & $34(40 \%)$ & $15(20 \%)$ \\
Mean rate change & $42(49 \%)$ & $14(19 \%)$ \\
Peak rate change & $35(41 \%)$ & $13(17 \%)$ \\
CoM change & $25(29 \%)$ & $6(8 \%)$ \\
Sparsity change & $35(41 \%)$ & $18(24 \%)$ \\
\hline
\end{tabular}

A resampling procedure was used to create rate maps with no spatial information and determine which cells had more spatial selectivity than expected by chance at the $p=0.05$ level. A trial-shuffling resampling procedure was used to determine which cells exhibited a significant amount of systematic rate map change across trials.

$p=1.0 \times 10^{-4}$, Fisher's exact test). Similarly, 58 of 75 GluA $1^{-1-}$ neurons had significantly greater sparsity than expected by chance, whereas 85 of 86 WT neurons did $\left(p=8.6 \times 10^{-6}\right)$. Thus, although the reductions in spatial selectivity of the 
GluA1 $1^{-/-}$neurons were substantial, a significant amount of spatial modulation remained in most $G l u A 1^{-/-}$neurons.

The low spatial selectivity of the $G l u A 1^{-/-}$rate maps often made it difficult to define clear place field boundaries. Hence, we quantified place field properties using two different definitions. First, we defined place fields as regions of space in which the spike rate exceeded $1 \mathrm{~Hz}$ for $\geq 10$ contiguous spatial bins $(5 \mathrm{~cm})$ (Muller et al., 1987; McHugh et al., 1996). The GluA1 ${ }^{-1-}$ cells had 1.6-fold more place fields per cell compared with WT neurons, but there was no significant difference between the size of individual place fields (Table 1). Because the peak spike rates of GluA1 $1^{-1-}$ neurons were 3.1-times lower than WT neurons, we used another adaptive method in which we defined place field boundaries as regions in which the spike rate exceeded $20 \%$ of the peak rate of the cell (Hollup et al., 2001; Kjelstrup et al., 2008). With this definition, there were 2.5 times as many place fields per cell for $\mathrm{GluA1}^{-/-}$neurons compared with WT (Fig. $2 E$ ), but individual place fields had similar widths in the two genotypes (Table 1). Moreover, GluA1 ${ }^{-1-}$ place cells had a greater tendency to spike outside of their place fields, as quantified by the reduced SNR of GluA1 $1^{-1-}$ place cells (Fig. $2 F$ ). These methods of quantifying the place fields of each cell are parameter sensitive, yet the results remain qualitatively valid for a range of parameters tested.

Because the spiking properties of hippocampal neurons depend on an animal's running speed (McNaughton et al., 1983; Wiener et al., 1989; Zhang et al., 1998; Muir and Bilkey, 2003), it is possible that the above results could be attributable to behavioral differences between WT and GluA1 ${ }^{-1-}$ mice. Across all sessions for which a recorded cell was used for analysis, the average running speeds (during epochs used for analysis) were slightly higher in the WT ( $n=52$ sessions; $20 \mathrm{~cm} / \mathrm{s}, 18-26 \mathrm{~cm} / \mathrm{s}$ ) than GluA1 $^{-/-}$mice ( $n=33$ sessions; $\left.18 \mathrm{~cm} / \mathrm{s}, 12-20 \mathrm{~cm} / \mathrm{s}\right)(p=$ $7.5 \times 10^{-3}$, Wilcoxon rank-sum test). However, this small behavioral difference could not explain the much larger differences in the spatial spiking properties of WT and $G l u A 1^{-/}$neurons. An ANCOVA revealed that, even after accounting for the differences in running speed, the differences between WT and GluA1 $1^{-1-}$ in all measures of spatial selectivity were still highly significant (see Materials and Methods).

\section{Differences in population vector encoding of space}

The above measures treated each cell separately, but information is thought to be encoded by the pattern of activity of populations of neurons (Georgopoulos et al., 1986; Wilson and McNaughton, 1993; Gothard et al., 1996; Leutgeb et al., 2005; Wills et al., 2005). Hence, we compared the spatial modulation of populations of WT and $G l u A 1^{-1-}$ neurons by computing the set of average spike rates across all recorded neurons (pooled across recording sessions) at each position, known as the population vector (Georgopoulos et al., 1986). The spike rate of each cell was normalized by its mean spike rate before performing population vector analysis so that all cells contributed approximately equally to the results (see Materials and Methods) (Battaglia et al., 2004), but all results were qualitatively similar without such normalization. Consistent with the differences in single-neuron spatial information, the matrix of population vectors showed clear spatial selectivity in the population of WT neurons (Fig. $3 A$ ) but not for $\mathrm{GluA1}^{-1-}$ neurons (Fig. 3B). As a result, the population vectors for WT were substantially more sparse than for $G l u A 1^{-/-}$across all spatial locations on the track, although both WT and $\mathrm{GluA1}^{-1-}$ population vectors were significantly more sparse than expected by chance (Fig. 3C).
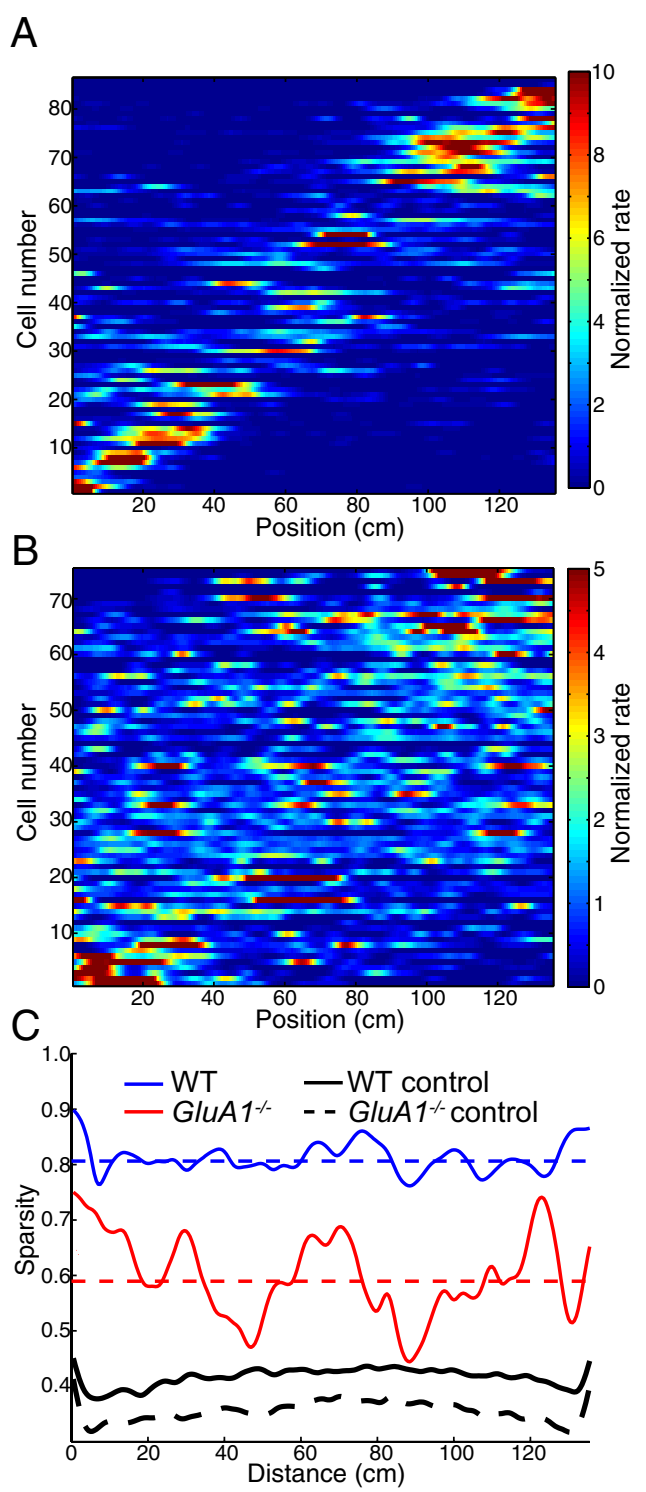

Figure 3. Reduced spatial modulation of $G l u A 1^{-/-}$population vectors. $A$, Matrix of WT population vectors for the rightward movement direction. Each row depicts the rate map of a neuron, normalized by the overall mean rate. The rate maps are sorted by the location of their center of mass. $\boldsymbol{B}$, Same as $\boldsymbol{A}$ for $G l u A 1^{-\prime-}$. C, Population vector sparsity as a function of position on the track (average of the two movement directions). The horizontal dashed blue and red lines show the median population vector sparsity for WT and GluA1 ${ }^{-/-}$, respectively the solid and dashed black lines show the expected sparsity of control population vectors in WT and $\mathrm{GluA1}^{-1-}$, respectively, having no spatial information. WT population vectors were significantly more sparse than GluA1 ${ }^{-1-}$ (WT, $0.80,0.78-0.84 ; \mathrm{GluA1}^{-1-}, 0.62,0.51-0.67 ; p=$ $1.8 \times 10^{-90}$, Wilcoxon signed-rank test).

The spatial tuning of the matrix of population vectors can be estimated by computing its autocorrelation function (Fig. 4) (Gothard et al., 1996; Battaglia et al., 2004; Kjelstrup et al., 2008). Because a majority of place cells are directional, the population vectors for the leftward and rightward journeys were treated separately. Points along the diagonal represent the correlations between each population vector with itself, which is unity. Points at increasing distances away from the diagonal represent the correlation, or similarity, between population vectors representing increasingly distant positions on the track. The population vector correlations for WT (Fig. 4A) and GluA1 ${ }^{-/-}$(Fig. 4B) both decayed rapidly with distance from the diagonal. The distance over which the population vectors (encoding the same movement di- 

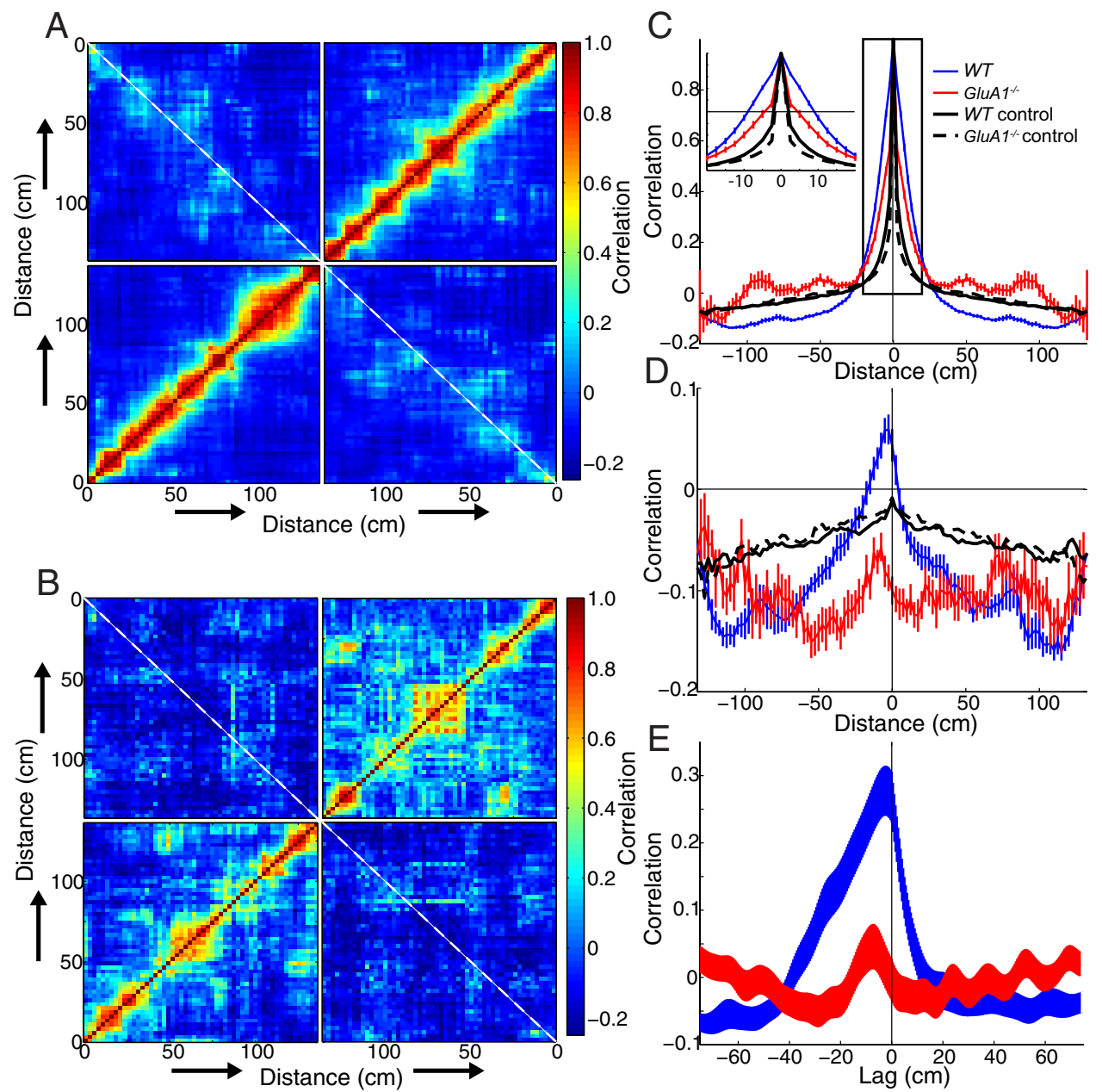

Figure 4. Altered population vector representation of position in $\mathrm{GluA1}^{-/-}$. A, Spatial autocorrelation matrix of the WT population vectors. The diagonal quadrants depict correlations of population vectors encoding the same movement direction, whereas off-diagonal quadrants show correlations for opposite movement directions. Dashed white lines along the off-diagonal quadrants represent correlations between identical absolute positions. Movement directions are indicated by the arrows. B, Same as $\boldsymbol{A}$ for $G l u A 1^{-1-}$. C, Average population vector correlation as a function of distance for population vectors encoding the same movement direction. The black solid and dashed lines show the population vector correlations for WT and GluA ${ }^{-1-}$ control population vectors with zero spatial information. The inset shows a magnified version of the region indicated by the black rectangle. The horizontal black line shows the $50 \%$ threshold used to define the decorrelation length. $\boldsymbol{D}$, Same as $C$ for population vectors encoding opposite movement directions. The prominent central peak in the WT indicates position-independent encoding of position in WT that is much less apparent in $\mathrm{GluA1}^{-1-}$. The location of the peak is at negative lags for both WT and $G l u A 1^{-1-}$, suggesting prospective coding. E, Average cross-correlograms of the single-neuron rate maps encoding opposite movement directions. Peaks at negative lags again indicate that the rightward rate maps are shifted to the left relative to leftward rate maps, suggesting prospective coding.

rection) become decorrelated provides a measure of the spatial scale of the population representation of space (Fig. 4C) (Battaglia et al., 2004; Kjelstrup et al., 2008). The half-width of the central autocorrelation peak (at 50\% of its maximal value) was approximately twice as large in WT $(9.0 \mathrm{~cm})$ compared with $G l u A 1^{-/-}$ $(4.6 \mathrm{~cm})$, although both were substantially wider than for the null controls (WT, $2.1 \mathrm{~cm}$; GluA1 ${ }^{-1-}, 1.7 \mathrm{~cm}$ ). Notably, to avoid the introduction of spurious structure to the population vector correlation matrix, these were computed using coarser spatial bins $(2.5 \mathrm{~cm})$ and no smoothing of the rate maps. Furthermore, for more spatially separated population vectors $(>20 \mathrm{~cm})$ encoding the same movement directions, the $G l u A 1^{-/-}$population vectors were significantly more correlated $(0.024,-0.029$ to 0.043$)$ than for WT $(-0.10,-0.12$ to 0.080$)$ ( $n=45$ unique separation distances; $p=6.8 \times 10^{-9}$, Wilcoxon signed-rank test). Thus, the WT population representation was much more spatially coherent than for $G l u A 1^{-/-}$, and population vectors encoding distant positions were more dissimilar.

The WT autocorrelation matrix (Fig. 4A) showed additional structure in the off-diagonal quadrants, corresponding to the overlap between population vectors encoding opposite movement directions. These off-diagonal quadrants showed clear diagonal bands indicating increased correlation between population vectors encoding similar positions on the opposite directions of the journey. Indeed, the average correlation as a function of distance revealed a clear central peak for WT that was significantly reduced in the GluA1-/- population (Fig. $4 D ; p=1.9 \times$ $10^{-5}$, two-sample $t$ test at the central peaks). This difference suggests there was a direction-independent representation of position in the WT population (Battaglia et al., 2004) that was only weakly present in the $G l u A 1^{-/-}$population. Moreover, the maximal correlation for WT population vectors occurred when the rightward population vector was shifted to the left of the leftward population vector on the track, which is suggestive of prospective coding (Muller and Kubie, 1989; Frank et al., 2000; Wood et al., 2000; Ferbinteanu and Shapiro, 2003; Battaglia et al., 2004; Pastalkova et al., 2008; Kennedy and Shapiro, 2009). Such prospective coding was slightly apparent in the $G l u A 1^{-/-}$population vector correlations as well, although to a much lesser extent. These relationships between the population vectors encoding opposite di- 
A
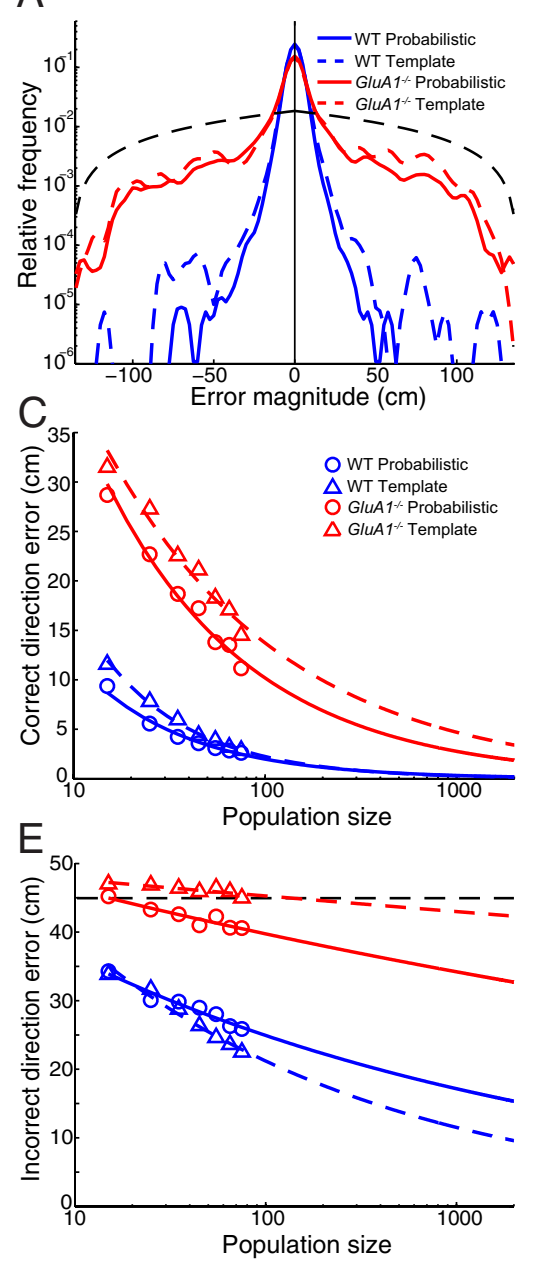

B
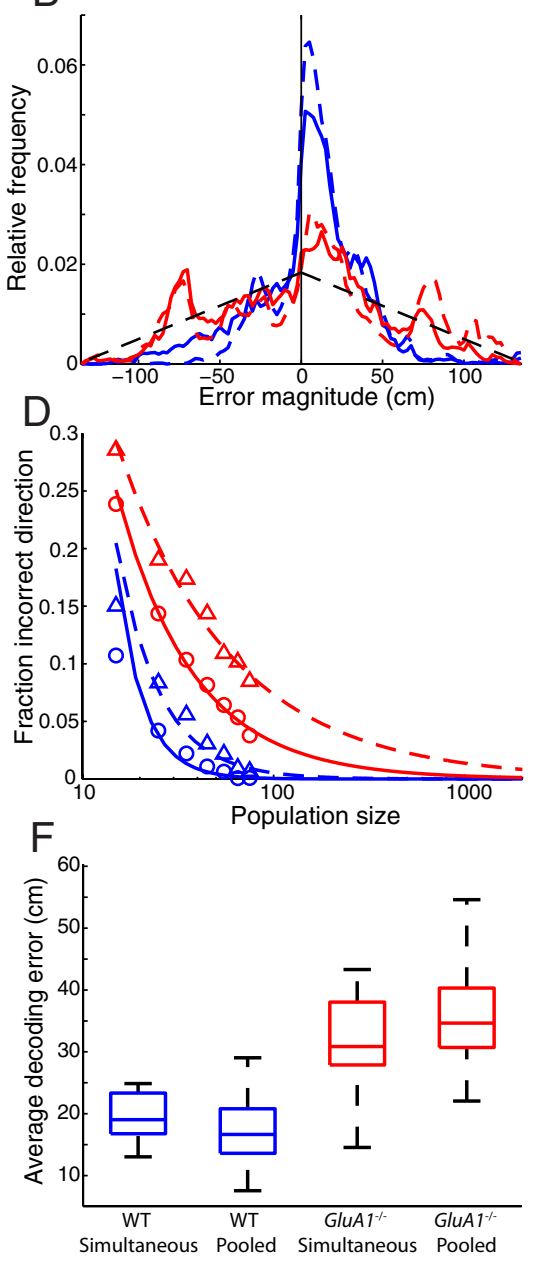

Figure 5. Reduced population decoding accuracy in $\mathrm{GluA}^{-/-}$. A, Distribution of decoding error magnitudes for WT and $\mathrm{GluA1}^{-1-}$ populations using probabilistic decoding and template matching. The black line shows the decoding error distribution for chance level. Same color conventions apply to $\boldsymbol{B}-\boldsymbol{E}$. $\boldsymbol{B}$, Distribution of decoding errors (relative to the movement direction) when the decoding algorithm is forced to choose among the positions encoding the opposite movement direction. The clear peak at positive errors for WT indicates that the algorithm tends to decode positions ahead of the animals' true position, or prospectively. The distribution of decoding errors for GluA1 ${ }^{-1-}$ is close to the distribution expected by chance (black line). C, Average decoding error is plotted for WT and $\mathrm{GluA} 1^{-/-}$as a function of population size. The circles and triangles depict errors for the probabilistic and template-matching algorithms, whereas the lines correspond to best-fit power law functions. Chance level decoding error is $45 \mathrm{~cm}$. $D$, The fraction of decoded positions that have the correct movement direction is plotted for WT and GluA1 ${ }^{-1-}$ as a function of population size. Chance level is 0.5 . $\boldsymbol{E}$, The average decoding error is plotted as a function of population size for forced oppositedirection decoding. $\boldsymbol{F}$, Average decoding errors were comparable for the largest simultaneously recorded populations of WT ( $n=$ $7 ; 19.0,16.8-23.3 \mathrm{~cm})$ and $\mathrm{GluA} 1^{-/-}(n=6 ; 30.9,27.9-38.1 \mathrm{~cm})$ neurons and random size-matched subsets of neurons pooled across recording sessions (WT, 16.7, 13.6-20.8 $\mathrm{cm}$; $\mathrm{GluA1}^{-1-}, 24.7,30.7-40.3 \mathrm{~cm}$ ).

rections were confirmed by computing cross-correlograms between the rightward and leftward rate maps for each cell separately. The average cross-correlogram for WT cells showed a prominent central peak that was shifted slightly (Fig. 4E), indicative of an absolute representation of position and prospective coding, respectively. Both of these features were greatly reduced for $G l u A 1^{-\prime-}$. Thus, although WT neurons were more directional overall than $G l u A 1^{-1-}$ neurons, those WT neurons that were active in both directions of the journey were more likely to be active in the same region of the track, although the same was not true for $\mathrm{GluA1}^{-/}$.

\section{Reduced accuracy of population decoding}

We next sought to estimate the spatial information content of populations of WT and $\mathrm{GluA1}^{-1-}$ place cells using a population

vector decoding analysis (Wilson and McNaughton, 1993; McHugh et al., 1996; Zhang et al., 1998). Specifically, we quantified how accurately the animals' position could be decoded from the single-trial spike rates of place cell populations using a leave-one-out crossvalidation procedure (Fig. 5; see Materials and Methods; Rutishauser et al., 2006). In each iteration, one randomly chosen trial for each cell was set aside as the test data, and the remaining trials were used to train the decoding algorithm. We used two different decoding procedures. The first was a simple template-matching method that compared the overlap between the singletrial and leave-one-out average (template) population vectors (Wilson and McNaughton, 1993; Zhang et al., 1998). The second was a probabilistic decoding procedure that used explicit models of the distributions of single-trial spike rates of each neuron as a function of position (Foldiak, 1992; Zhang et al., 1998; Pouget et al., 2000). The decoding accuracy was measured in two ways: the probability of decoding the incorrect movement direction, and the difference between the decoded position and the actual position given the movement direction was correctly decoded.

As expected, given the differences in WT and $G l u A 1^{-1-}$ single-neuron spatial information, decoding based on equivalently sized (75 neurons) populations of WT neurons was far more accurate than for $\mathrm{GluA1}^{-1-}$ populations (Fig. 5A; average decoding errors: WT probabilistic, $2.6,2.3-2.9 \mathrm{~cm}$; WT template-matching, $2.9,2.6-3.3 \mathrm{~cm} ; \mathrm{GluA1}^{-1-}$ probabilistic, $11.2,9.4-13.2 \mathrm{~cm}$; $\mathrm{GluA1}^{-/-}$templatematching, $14.2,12.0-16.8 \mathrm{~cm})$. Large decoding errors $(>20 \mathrm{~cm})$ almost never occurred with WT populations (probabilistic, $0.14 \%$; template-matching, $0.44 \%$ ), whereas they occurred $\sim 15 \%$ of the time for $\mathrm{GluA1}^{-\prime-}$ populations (probabilistic, $14 \%$; template-matching, $17 \%$ ). Similarly, the probability of decoding the incorrect movement direction was much higher for GluA1 ${ }^{-1-}$ (probabilistic, 3.8\%; template-matching, $8.3 \%$ ) compared with WT (probabilistic, $0.13 \%$; template-matching, $0.78 \%)$ populations. Despite these relatively large reductions in the spatial information contained in the $G l u A 1^{-1-}$ population, the decoding was still far more accurate than would be expected by chance $(70 \%$ of errors $>20 \mathrm{~cm}$, and 50\% incorrect movement direction; Fig. 5A, black dashed line). This result is consistent with the observation that a majority of $\mathrm{GluA1}^{-1-}$ neurons retained a significant amount of spatial information (Table 2). To quantify the direction-independent population representation of position, we also computed population decoding errors after forcing the algorithm to choose among the positions along the opposite direction of the journey. Consistent with the population vector autocorrelation analysis, 
WT decoding accuracy for the opposite movement direction was much better than chance, whereas $G l u A 1^{-1-}$ was near chance level (Fig. 5B; average decoding errors: WT probabilistic, 26.0, 23.8-28 cm; WT template-matching, 21.9, 20.1-23.9 cm; GluA1 $1^{-/-}$probabilistic, $41.1,38.8-43.4 \mathrm{~cm}$; GluA1 $1^{-/-}$templatematching, $46.6,43.0-50.1 \mathrm{~cm})$. Interestingly, the templatematching algorithm performed significantly better than the probabilistic method for the WT, but the converse was true for the GluA1 $1^{-/-}$population. This difference suggests that, in the WT, the relative pattern of spike rates across the population, rather than their absolute values, provide a better representation of absolute position, whereas the converse is true for the GluA1 $1^{-1-}$.

Because the relevant population of CA1 neurons is likely to be much larger than the number recorded here $[\sim 200,000$ total pyramidal neurons (Calhoun et al., 1998)], it is possible that the difference in WT and GluA1 ${ }^{-1-}$ decoding accuracy would become negligibly small for sufficiently large populations of neurons. To estimate the dependence of decoding accuracy on population size, we randomly subsampled the WT and GluA1 ${ }^{-/-}$ populations and measured the decoding accuracy in terms of both error magnitude (Fig. 5C) and the probability of decoding the incorrect movement direction (Fig. 5D). This analysis revealed that the relative difference between WT and GluA1 ${ }^{-1-}$ decoding accuracy decreased with population size, and the WT decoding accuracy began to saturate at an accuracy of $\sim 2 \mathrm{~cm}$. To estimate the decoding accuracy of larger populations, we extrapolated from our data using best-fit power law relationships (see Materials and Methods). Indeed, $G l u A 1^{-/-}$populations of only a few hundred neurons are predicted to have average decoding errors $<5 \mathrm{~cm}$ and incorrect direction decoding $<1 \%$ of the time. Thus, although the decoding accuracy of the measured populations of neurons was much smaller for WT than $G l u A 1^{-1-}$, the GluA $1^{-1-}$ populations performed much better than chance and are predicted to converge to similar levels of decoding accuracy for larger population sizes. We also quantified the accuracy of opposite-direction decoding as a function of population size and found that, although decoding accuracy improved robustly with increasing population size for WT (particularly using the template-matching algorithm), improvements were far more modest for GluA1 ${ }^{-/-}$(Fig. $5 E$ ). Thus, the large reduction in the accuracy of the GluA1 $1^{-1-}$ population compared with WT in representing absolute position, independent of movement direction, is predicted to remain even for populations of several thousand neurons.

Although populations of neurons were pooled across recording sessions for the population decoding analysis used here (see Materials and Methods), we also performed a decoding analysis using the largest simultaneously recorded populations of WT (seven neurons) and GluA1 ${ }^{-/-}$(six neurons) for comparison. Average decoding errors were comparable for simultaneously recorded and pooled populations of both genotypes (Fig. 5F), suggesting that pooling neurons did not substantially affect our results. This is perhaps to be expected given that studies performing population decoding using simultaneously recorded populations explicitly or implicitly assume independent coding of position among place cells (Wilson and McNaughton, 1993; Zhang et al., 1998).

\section{Altered burst patterns}

The average ISI distributions across the populations of WT and GluA1 $1^{-1-}$ cells showed substantial differences in several distinct ISI ranges (Fig. 6A). To quantify these differences, we divided the
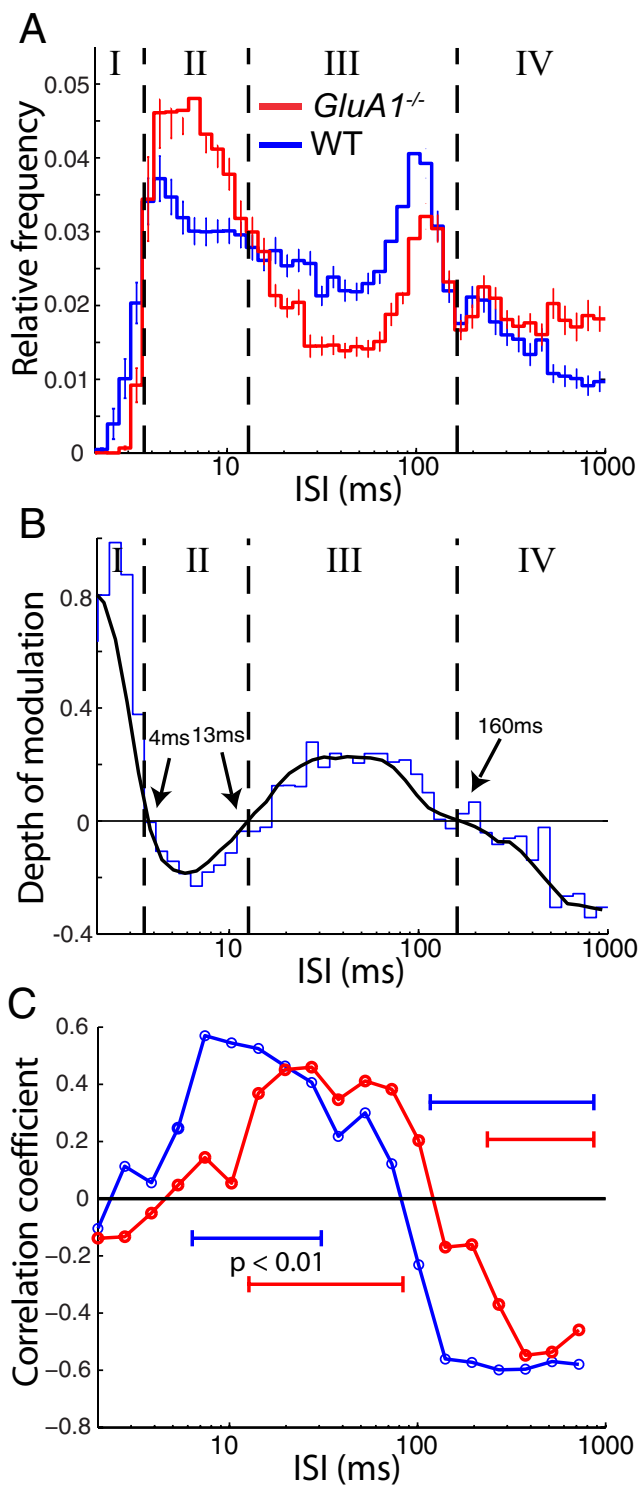

Figure 6. Altered ISI distributions in GluA1 ${ }^{-/-}$neurons. A, Average ISI distributions for WT and $G / u A 1^{-/-}$neurons (during trial running). The vertical dashed lines indicate the boundaries between the four ISI regions of interest, at 4, 13, and $160 \mathrm{~ms}$. B. The depth of modulation of the $\mathrm{GluA1}^{-1-}$ ISI distribution (relative to the WT) is computed in each ISI bin (blue trace) and smoothed slightly (black trace). The zero crossings of the smoothed depth of modulation are used to define the four ISI regions of interest indicated by the vertical dashed lines. $C$, The correlation coefficient between ISI probability and spatial information content is computed across WT and GluA1 ${ }^{-1-}$ cells for 20 logarithmically spaced ISI bins. Ranges of ISI bins with significant correlations (at the $p=0.01$ level) are indicated by the blue and red horizontal lines for WT and $\mathrm{GluA1}^{-1-}$, respectively.

range of ISIs $(<1 s)$ into four non-overlapping intervals (see Materials and Methods; Fig. 6B). Although the WT neurons had a significantly larger fraction of very short ( $<4 \mathrm{~ms}$ ) ISIs (WT, 4.8, $1.4-7.8 \% ;$ GluA1 $\left.^{-1-}, 1.1,0-5.2 \% ; p=2.6 \times 10^{-4}\right)$, the GluA1 $1^{-1-}$ neurons had a substantially greater fraction of ISIs in the range $4 \mathrm{~ms}<\mathrm{ISI}<13 \mathrm{~ms}$ (WT, 27, 21-32\%; GluA1 ${ }^{-/-}$, 37, $\left.27-44 \% ; p=4.4 \times 10^{-7}\right)$. Thus, although WT neurons were able to achieve higher intra-burst frequencies, the GluA1 $1^{-1-}$ neurons had $22 \%$ more burst ISIs $\left(<13 \mathrm{~ms} ; p=3.6 \times 10^{-3}\right)$ than WT. Additionally, WT neurons were significantly more likely to have ISIs in the intra-theta cycle range $13 \mathrm{~ms}<$ ISI $<160 \mathrm{~ms}$ (WT, 49, 39-58\%; GluA1 $\left.1^{-1-}, 36,28-48 \% ; p=2.4 \times 10^{-8}\right)$, whereas 
A
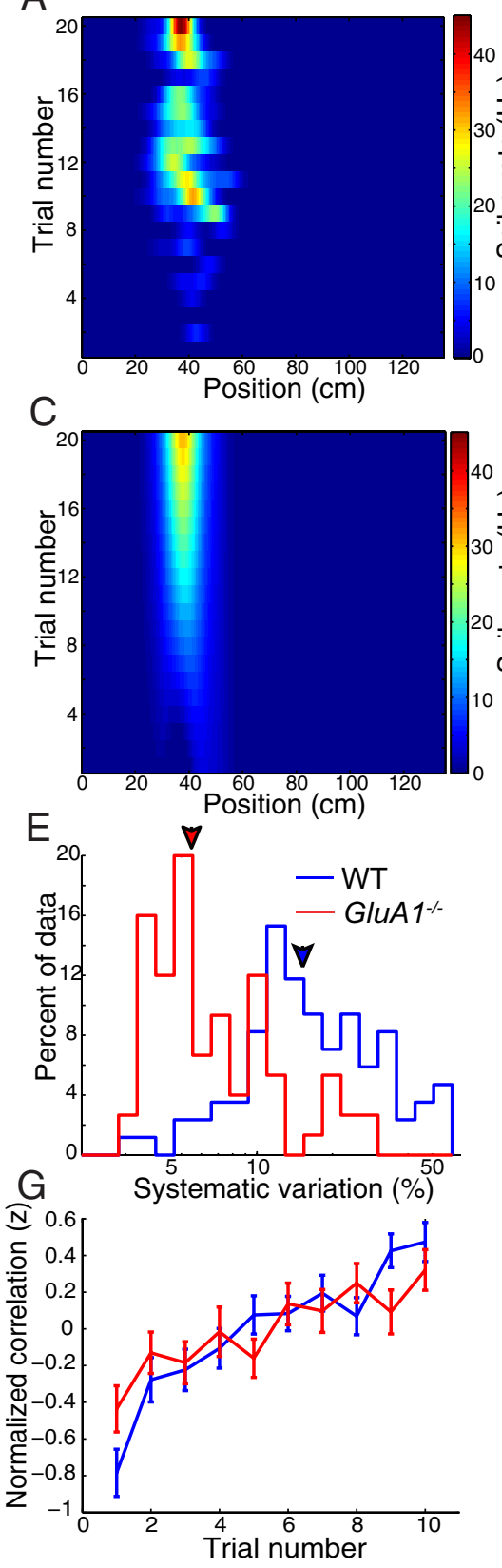

B
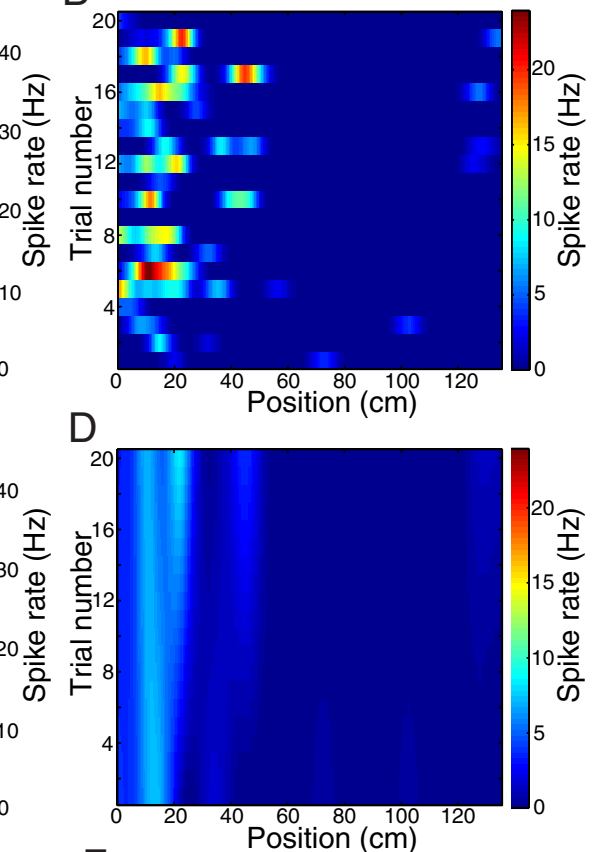

$\mathrm{F}$
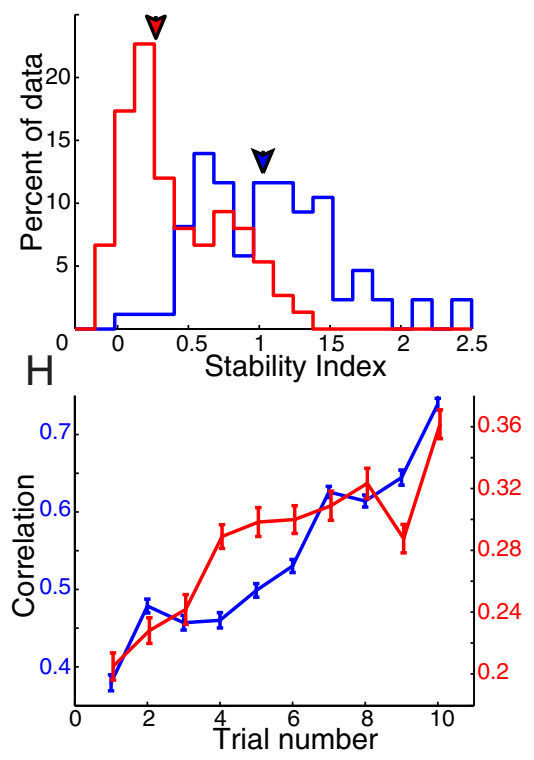

Figure 7. Impaired rate map plasticity and stability in $\mathrm{GluA1^{-/- }}$. $\boldsymbol{A}$, Trial-by-trial rate map matrix for an example WT neuron. Each row depicts the rate map in a single trial, with trial number increasing from bottom to top. $\boldsymbol{B}$, Same as $\boldsymbol{A}$ for an example $G l u A 1^{-/-}$neuron. $C$, Matrix showing the linear fit of the single-trial spike rate at each position as a function of trial number for the data shown in $\boldsymbol{A}$. D, Same as $\left(\right.$ for the GluA $1^{-/-}$neuron. $\boldsymbol{E}$, Distributions of the systematic variation in the single-trial rate maps computed for the populations of WT and GluA1 ${ }^{-/-}$neurons. WT neurons had more systematic rate map change (15\%, 11-26\%) than $\mathrm{GluA1}^{-/-}\left(5.5 \%, 4.2-10 \% ; p=9.6 \times 10^{-14}\right) . \boldsymbol{F}$, Distributions of the trial-by-trial rate map stability index. Rate map stability was higher in WT $(1.03,0.67-1.4)$ compared with GluA1 $^{-/-}\left(0.27,0.13-0.74 ; p=3.9 \times 10^{-15}\right)$. G, The correlation between single-trial rate maps and the lookup rate map (computed over trials 11-15) is plotted as a function of trial number. Correlations for each neuron are normalized to units of $z$-score across trials for direct comparison of systematic changes across trials. Correlation coefficients between the lookup correlation and trial number were significantly positive for both WT $(0.41,0.05-0.68$; $\left.p=1.5 \times 10^{-7}\right)$ and $\mathrm{GluA1}^{-/-}\left(0.19,-0.11\right.$ to $\left.0.41 ; p=2.9 \times 10^{-4}\right)$. $\boldsymbol{H}$, The correlation between single-trial population vectors and lookup population vectors is plotted as a function of trial number. The correlation coefficient between trial number and this lookup correlation was large and highly significant for both WT $\left(r=0.96, p=1.4 \times 10^{-5}\right)$ and GluA ${ }^{-/-}(r=0.90, p=$ $4.3 \times 10^{-4}$ ). Note the different $y$-axes.

GluA1 $1^{-/-}$neurons were significantly more likely to show very long ISIs (>160 ms; WT, 16, 9.5-23\%; GluA1 ${ }^{-/-}, 22,17-29 \%$; $\left.p=2.1 \times 10^{-4}\right)$. To determine whether these changes in ISI distributions of $G l u A 1^{-/-}$neurons were related to the differences

in spatial selectivity between genotypes, we computed the correlation coefficient between ISI probability and spatial information content. Interestingly, the spatial information content of a neuron was strongly correlated with its ISI distribution for both WT and GluA1 $1^{-/-}$neurons (Fig. 6C). More spatially informative neurons had significantly more shortduration ISIs $(p<0.01$ for WT ISIs $7-27$ $\mathrm{ms}$; GluA1 ${ }^{-/-}, 14-73 \mathrm{~ms}$ ) and fewer longduration ISIs (WT, 195-1000 ms; GluA1 ${ }^{-/-}$, 375-1000 ms). Thus, neurons that achieved higher maximal firing rates over very short periods, i.e., had stronger bursting, also tended to be more spatially informative. This suggests that neurons that received stronger excitatory drive had more spatially selective spiking. Conversely, reduced burst frequencies, peak rates, and spatial information in GluA $1^{-/-}$ neurons might all be attributable to a decreased excitatory drive.

\section{Reduced experience-dependent changes} in activity and reduced trial-by-trial rate map stability

The experiential dynamics of place cell activity, including stability (Rotenberg et al., 1996; Kentros et al., 1998) and plasticity (Blum and Abbott, 1996; Mehta et al., 1997, 2000; Lee et al., 2004), are thought to be important for spatial reference memory. Rate map plasticity refers to systematic changes with experience in the spatial spiking properties of place cells (Mehta et al., 1997, 2000; Lee et al., 2004), as well as head direction cells (Yu et al., 2006), that occur on timescales as fast as single trials and that are thought to be required for spatial learning (Blum and Abbott, 1996; Mehta et al., 2000). Conversely, variability in the single-trial rate maps that is not related to experience is thought to impair the maintenance of stable and accurate representations of position (Kentros et al., 1998). Because most GluA1 ${ }^{-/-}$ neurons did not have clearly defined place fields, we quantified the across-trial dynamics of the entire spike rate maps rather than of individual place fields.

Figure 7, $A$ and $B$, shows the trial-bytrial rate map matrices for example wellisolated WT and $G l u A 1^{-/-}$place cells. The WT neuron exhibited a pronounced and systematic increase in spike rate across trials (correlation between average spike rate and trial number, $r^{2}=0.52, p=$ $\left.3.6 \times 10^{-4}\right)$, whereas the spike rate of the GluA1 $1^{-1-}$ neurons did not show a significant systematic change $\left(r^{2}=0.04, p=0.40\right)$. To quantify the amount of systematic variation in the rate maps, we fit a linear model of spike rate as a function of trial number at each spatial bin (Fig. 7C,D). The percentage of the total across-trial variance 
in the rate map matrix of each neuron explained by the linear model was then taken as a measure of rate map plasticity, termed "systematic variation" (see Materials and Methods). For the example WT neuron, nearly half of the total variance (47\%) was attributable to systematic across-trial changes, whereas for the GluA1 $1^{-/-}$neuron, the systematic variation was only $4 \%$. It is also evident from Figure 7, $A$ and $B$, that the spatial distribution of spiking was far more stable (less trial-by-trial variability) for the WT neuron compared with the GluA1 $1^{-/-}$neuron. This was confirmed by computing the stability index (see Materials and Methods), which was substantially higher for the WT neuron (1.5) compared with the GluA1 $1^{-/-}$neuron $\left(0.91 ; p=3.0 \times 10^{-3}\right.$, Wilcoxon rank-sum test).

The indications from these example neurons were confirmed by computing these measures of plasticity and stability for all neurons (Table 1). Indeed, there was a 2.8 -fold decrease in the systematic variation of the GluA1 $1^{-/-}$rate maps compared with WT (Fig. 7E). We used a trial-shuffling resampling procedure to determine the fraction of neurons from each genotype that showed a significant amount of systematic variation. Although a much lower proportion of GluA1 $1^{-/-}$neurons (13 of 75) had significant systematic variation compared with WT (55 of 86), this fraction of $G l u A 1^{-/-}$neurons was still significantly more than expected by chance ( $p=8.3 \times 10^{-5}$, binomial test; Table 2$)$. In addition to this global measure of systematic rate map change, we also quantified the systematic change in specific rate map properties, such as the mean rate, peak rate, COM, and sparsity (Table 1; see Materials and Methods). Consistent with the differences in overall systematic variation, neurons in WT mice showed more systematic change in each of these rate map statistics than GluA1 ${ }^{-1-}$ (Table 1), yet more $G l u A 1^{-/-}$neurons had significant systematic change than expected by chance (Table 2). In addition to the large reduction in rate map plasticity, GluA1 $1^{-1-}$ neurons also exhibited a 3.8-fold decrease in rate map stability (Fig. $7 F$ ). Thus, GluA1 ${ }^{-/-}$rate maps exhibited less systematic change across trials and had a much higher degree of random trial-by-trial variability than their WT counterparts.

The above measures of plasticity are particularly sensitive to overall changes in spike rate but are less sensitive to changes in the relative spatial distribution of a neuron's spiking. To quantify any systematic changes in the spatial distribution of the rate map of each neuron (termed the "rate distribution") across trials, we computed the overlap (Pearson's correlation coefficient) between each single-trial rate map and a "lookup" rate map generated by averaging over the final five trials (see Materials and Methods). This measure is insensitive to changes in the overall spike rate across trials and will increase across trials for neurons with systematically changing rate distributions. Consistent with the previous analyses, WT rate distributions showed 2.1-fold greater changes with experience than $G l u A 1^{-1-}$, but both WT and $G l u A 1^{-1-}$ rate distributions showed significant amounts of systematic change (Fig. 7G; WT, $p=1.5 \times 10^{-7}$; $G l u A 1^{-/-}, p=$ $2.9 \times 10^{-4}$; Wilcoxon signed-rank tests). As another test of systematic changes in spatial spiking properties, we computed the similarity of single-trial population vectors and those computed from the lookup rate maps as a function of trial number (Fig. $7 \mathrm{H}$; see Materials and Methods). The correlation coefficient between trial number and the population vector lookup correlation was large and highly significant for both WT $(r=0.96, p=1.4 \times$ $\left.10^{-5}\right)$ and $\mathrm{GluA1}^{-1-}\left(r=0.90, p=4.3 \times 10^{-4}\right)$, again indicating that significant systematic rate map changes occurred in the GluA1 $1^{-1-}$ neurons, although they were greatly reduced relative to WT neurons.
To test whether the observed systematic changes in the rate maps of WT and GluA1 ${ }^{-/-}$neurons could be explained by systematic changes in behavior across trials, particularly changes in running speed, we performed ANCOVA (see Materials and Methods). Using the correlation coefficient between running speed and trial number as a metric of behavioral change, we tested whether differences in WT and $G l u A 1^{-/-}$rate map changes could be accounted for by differential behavioral changes. Interestingly, WT mice significantly slowed down across trials ( $n=52$ unique sessions; $r=-0.36,-0.49$ to $0.14 ; p=$ $2.4 \times 10^{-6}$, Wilcoxon's signed-rank test), whereas GluA1 $1^{-/-}$ mice did not show any significant changes in running speed across trials $(n=33 ; r=0.12,-0.36$ to $0.46 ; p=0.41)$. However, such behavioral changes across trials were uncorrelated with the amount of systematic rate map change (WT, $r=0.11, p=0.32$; GluA1 $1^{-/-}, r=-0.087, p=0.46$ ) or the stability index (WT, $r=$ $-0.12, p=0.25$; GluA1 $^{-/-}, r=-0.18, p=0.12$ ). An ANCOVA revealed that across-trial changes in speed were significantly correlated with changes in the rate distribution $\left(p=7.1 \times 10^{-3}\right)$, and this correlation was found to account for the difference between WT and $\mathrm{GluA1}^{-1-}$ rate distribution changes $(p=0.45)$. However, the amount of systematic rate distribution change was still significantly greater than 0 for both WT $\left(p=1.4 \times 10^{-5}\right)$ and GluA1 $^{-1-}\left(p=5.3 \times 10^{-4}\right)$, even after accounting for the correlation between rate distribution change and behavioral change (see Materials and Methods). Although we cannot rule out that behavioral changes that were not well described by measured parameters could explain away the observed changes in rate maps and rate distributions, similar results were obtained using other measures of single-trial behavior, including the number of stops per trial, the time spent stopped per trial, and the average single-trial speed after excluding stops and pauses (speed $<5$ $\mathrm{cm} / \mathrm{s}$ ) (data not shown).

\section{Discussion}

These results demonstrate that the removal of GluA1-containing AMPARs dramatically reduced all measures of the spatial selectivity of CA1 neurons. Although these deficits were comparable with, or greater than, those reported in several other transgenic studies (McHugh et al., 1996; Rotenberg et al., 1996; Korotkova et al., 2010; Allen et al., 2011), novel analyses revealed that a significant amount of spatial selectivity still remained. This was true at the level of both the spatial spiking properties of individual neurons and the population representation of position. These results were confirmed with a population decoding analysis, using two different decoding methods. Although the accuracy of the hippocampal population code for space in $G l u A 1^{-1-}$ mice was much less than that of WT for small neural populations, for larger more biologically relevant populations, GluA $1^{-/-}$decoding accuracy was predicted to be similar to that of WT. In addition, the $G l u A 1^{-/-}$ population exhibited a near complete elimination of the direction-independent representation of position observed in the WT population, despite the reduced directionality of $G l u A 1^{-/-}$ neurons. This large reduction of the direction-independent representation of space in $G l u A 1^{-/-}$was predicted to remain even for biologically relevant population sizes.

Moreover, although the GluA1 $1^{-1-}$ neurons had poor spatial selectivity, which precludes the usual analysis of place field plasticity (Mehta et al., 1997, 2000; Lee et al., 2004), novel analysis methods revealed that the rate maps of GluA1 $1^{-/-}$neurons showed greatly reduced, but significantly greater than chance, levels of systematic change across trials. At the same time, $G l u A 1^{-1-}$ neurons also showed substantial increases in the 
amount of random trial-by-trial variability in their rate maps. Surprising alterations were also observed in the temporal structure of $G l u A 1^{-/-}$neurons, spiking such that, despite slightly reduced excitatory transmission attributable to the removal of GluA1 (Andrásfalvy et al., 2003; Jensen et al., 2003; Romberg et al., 2009), the GluA1 ${ }^{-/-}$place cells were more likely to spike in bursts than WT, although they had lower peak intra-burst frequencies.

What insights do these results provide about the cellular mechanisms governing place cell activity? The alterations in both synaptic plasticity (Hoffman et al., 2002; Frey et al., 2009) and the dendritic distribution of synaptic strengths (Andrásfalvy et al., 2003; Jensen et al., 2003; Romberg et al., 2009) in the GluA1 $1^{-/-}$neurons could be responsible for the observed deficits in CA1 spatial selectivity. Surprisingly, manipulations that produce more dramatic alterations of the physiological forms of LTP, such as genetic activation of CaMKII (Rotenberg et al., 1996), application of NMDA blocker (Ekstrom et al., 2001), or genetic silencing of NMDARs within CA1 (McHugh et al., 1996) and deeper cortical layers (Fukaya et al., 2003; Rondi-Reig et al., 2006; Wiltgen et al., 2010) have been shown to produce comparable, or even less pronounced, effects on CA1 spatial selectivity. Similarly, manipulations that produce much larger perturbations of synaptic inputs to CA1, such as through complete lesion of CA3 inputs (Brun et al., 2002), genetic inactivation of CA3 (Nakashiba et al., 2008), or lesion of direct entorhinal inputs (Brun et al., 2008), have been observed to produce relatively mild impairments of CA1 spatial selectivity. Thus, it is possible that both factors, altered synaptic plasticity and the altered distribution of synapse strength on a dendrite, together contribute to the large impairments of spatial selectivity in $G l u A 1^{-/-}$place cells.

Because the impairments in more physiologically relevant forms of plasticity are minimal in GluA1 $1^{-1-}$ mice, we propose a simple mechanism to explain the alterations of spatial and temporal spiking properties of $G l u A 1^{-/-}$place cells based only on the reduced excitatory drive onto CA1 pyramidal neurons (Andrásfalvy et al., 2003; Jensen et al., 2003; Romberg et al., 2009) and interneurons (Fuchs et al., 2007) of adult animals. Specifically, we suggest that the reduced frequency of intra-burst spiking in GluA1 $1^{-1-}$ pyramidal neurons reflects the reduced excitatory drive, whereas the increased overall burst probability of $G l u A 1^{-/-}$neurons is attributable to the impaired recruitment of recurrent inhibition that normally regulates bursting in these neurons. This reduced activation of recurrent inhibition within CA1 could also explain the decreased sparsity of rate maps and population vectors in $G l u A 1^{-/-}$mice because of the impairment of competitive interactions among place cells. Such a relationship between excitatory drive and spatial information is supported by our observation that neurons with more short-duration ISIs, indicative of greater excitatory drive, had more selective rate maps in both WT and $G l u A 1^{-/-}$. In fact, similar reductions in the spatial selectivity of hippocampal place cells have been observed in mice lacking NMDARs in parvalbumin-expressing interneurons (Korotkova et al., 2010), and a similar increase in burstiness was observed in the CA1 neurons of mice lacking GluA1 in these interneurons (Rácz et al., 2009). Both of these manipulations reduced the excitatory drive onto inhibitory hippocampal interneurons. In a simple scenario, this would result in reduced inhibition onto excitatory neurons and hence a net increase in excitation. However, these studies did not report an increase in peak firing rates. Hence, not only the net excitatory drive onto the excitatory neurons, but the precise timing of excitatory and inhibitory inputs may be crucial in determining the peak firing rates and spatial selectivity of place cells (Ahmed and Mehta, 2009).

We show that the GluA1 $1^{-/-}$place cells exhibited major reductions in the amount of systematic change in their rate maps across trials, a process that is thought to be important for spatial learning (Blum and Abbott, 1996; Mehta et al., 2000). Although some significant experience-dependent changes were still observed in the rate maps of GluA1 $1^{-1-}$ neurons, the strong reductions in such rate map plasticity are especially surprising given that it is thought to be mediated by STDP (Mehta et al., 2000), which is intact in the GluA1 $1^{-1-}$ mice (Frey et al., 2009). Although it is possible that other GluA1dependent forms of synaptic plasticity are also required for such experience-dependent changes, we propose that, in GluA1 $1^{-1-}$ mice, the reduced trial-by-trial stability of rate maps could result in more variable coactivation and timing of presynaptic and postsynaptic neurons and thus lead to reductions in rate map plasticity across trials.

We note that the GluA1 subunit is deleted in the entire brain in these mice, and thus the observed changes in the activity of CA1 neurons could arise in afferent structures, such as the entorhinal cortex, in which neurons known as grid cells show multiple, spatially periodic place fields (Fyhn et al., 2004; Hafting et al., 2005; Yartsev et al., 2011). However, we focus our attention on CA1 because alterations of synaptic strength and LTP have been demonstrated only in CA1 (Zamanillo et al., 1999; Andrásfalvy et al., 2003; Jensen et al., 2003; Frey et al., 2009; Romberg et al., 2009), and the GluA1 subunit has highest expression within the hippocampus (PellegriniGiampietro et al., 1991). In fact, LTP is unaltered in the neocortex of $\mathrm{GluA1}^{-/-}$mice (Frey et al., 2009), suggesting minimal disruption of extrahippocampal circuits. Furthermore, juvenile mice display GluA1-independent LTP, which could generate intact wiring of hippocampal circuits in GluA1 ${ }^{-1-}$ mice during development (Jensen et al., 2003). Thus, altered spatial selectivity of $G l u A 1^{-/-}$hippocampal neurons is unlikely to arise during development and likely occurs during experience on the maze. Accordingly, conditional restoration of GluA1 receptors in adult $G l u A 1^{-/-}$mice rescues both synaptic and behavioral deficits (Mack et al., 2001; Schmitt et al., 2005). Furthermore, a significant amount of spatial selectivity develops even when neonatal animals, with immature neocortices, explore the world for the first time (Langston et al., 2010; Wills et al., 2010). Hippocampal spontaneous activity patterns are also predictive of the subsequent place cell activity in a novel environment (Dragoi and Tonegawa, 2011), and continually changing activity patterns even occur when rats run on a stationary wheel placed on a maze (Pastalkova et al., 2008). These studies suggest that a significant amount of hippocampal spatial selectivity is "hardwired," via $G l u A 1^{-1-}$ independent mechanisms, which could explain the significant remaining spatial selectivity in the GluA1 $1^{-/-}$ mice. The mechanisms of this hardwiring are unknown, but one possibility is that the unit of spatial memory is not a synapse but a sequence of synapses on a dendritic branch, formed by spontaneously generated dendritic spikes (Mehta, 2004), which could generate place field-like sequential activity under a variety of conditions listed above and in the GluA1 ${ }^{-/-}$ mice. Alternately, mechanisms based on inhibition could generate limited spatial selectivity in regions with relatively lower 
inhibition (Ahmed and Mehta, 2009). Additional experiments are needed to decipher this puzzle.

The relationships between CA1 activity and behavior appear similarly complex. Because the $G l u A 1^{-1-}$ mice are impaired on various tests of spatial working memory but not spatial reference memory (Zamanillo et al., 1999; Reisel et al., 2002; Schmitt et al., 2003), our results suggest that normal levels of CA1 spatial selectivity, rate map stability, and rate map plasticity are not likely to be required for reference memory tasks, such as the Morris water maze. This is consistent with recent experiments showing normal water maze performance despite reductions in CA1 spatial selectivity (Korotkova et al., 2010; Allen et al., 2011) and stability (Allen et al., 2011). Our results do suggest that these properties of CA1 activity may be required for normal spatial working memory, but it is unclear which properties in particular are necessary. One possible explanation is that reference memory is less sensitive than working memory to perturbations of hippocampal spatial selectivity (but see Sanderson et al., 2009), and that the remaining spatial modulation in $\mathrm{GluA1}^{-1-}$ neurons is sufficient to mediate normal reference memory performance in these animals. This is supported by the observation that, despite dramatic reductions in spatial selectivity, significant spatial information was still present in a majority of $G l u A 1^{-1-}$ neurons, and the population decoding accuracy was still far better than chance level. An analysis of decoding accuracy as a function of population size suggested that, for population sizes likely to be biologically relevant ( $>1000$ neurons), the differences between WT and $G l u A 1^{-1-}$ are expected to become negligibly small. This, combined with the significant residual rate map plasticity in $G l u A 1^{-/}$neurons, could allow the $G l u A 1^{-/-}$mice to learn a fixed spatial location, as in spatial reference memory tasks. Together with previous studies, our results suggest that the impaired experience-dependent plasticity and trial-by-trial stability of the $\mathrm{GluA1}^{-1-}$ neurons, rate maps in particular could lead to the impaired working memory performance seen in these animals, potentially because working memory tasks require a flexible representation of position that is rapidly modified with trial-specific information. The altered population representation of position, in particular the lack of a direction-independent representation of position in the GluA1 ${ }^{-/-}$population, could also contribute to the behavioral impairment.

\section{References}

Ahmed OJ, Mehta MR (2009) The hippocampal rate code: anatomy, physiology and theory. Trends Neurosci 32:329-338.

Aitchison J (1955) On the distribution of a positive random variable having a discrete probability mass at the origin. J Am Stat Assoc 50:901-908.

Allen K, Fuchs EC, Jaschonek H, Bannerman DM, Monyer H (2011) Gap junctions between interneurons are required for normal spatial coding in the hippocampus and short-term spatial memory. J Neurosci 31:6542-6552.

Andrásfalvy BK, Smith MA, Borchardt T, Sprengel R, Magee JC (2003) Impaired regulation of synaptic strength in hippocampal neurons from GluR1-deficient mice. J Physiol 552:35-45.

Bach ME, Hawkins RD, Osman M, Kandel ER, Mayford M (1995) Impairment of spatial but not contextual memory in CaMKII mutant mice with a selective loss of hippocampal LTP in the range of the theta frequency. Cell 81:905-915.

Battaglia FP, Sutherland GR, McNaughton BL (2004) Local sensory cues and place cell directionality: additional evidence of prospective coding in the hippocampus. J Neurosci 24:4541-4550.

Blum KI, Abbott LF (1996) A model of spatial map formation in the hippocampus of the rat. Neural Comput 8:85-93.

Britten KH, Shadlen MN, Newsome WT, Movshon JA (1992) The analysis of visual motion: a comparison of neuronal and psychophysical performance. J Neurosci 12:4745-4765.

Brun VH, Otnass MK, Molden S, Steffenach HA, Witter MP, Moser MB, Moser EI (2002) Place cells and place recognition maintained by direct entorhinal-hippocampal circuitry. Science 296:2243-2246.

Brun VH, Leutgeb S, Wu HQ, Schwarcz R, Witter MP, Moser EI, Moser MB (2008) Impaired spatial representation in CA1 after lesion of direct input from entorhinal cortex. Neuron 57:290-302.

Buzsáki G (1986) Hippocampal sharp waves: their origin and significance. Brain Res 398:242-252.

Buzsáki G, Eidelberg E (1983) Phase relations of hippocampal projection cells and interneurons to theta activity in the anesthetized rat. Brain Res 266:334-339.

Buzsáki G, Horváth Z, Urioste R, Hetke J, Wise K (1992) High-frequency network oscillation in the hippocampus. Science 256:1025-1027.

Calhoun ME, Kurth D, Phinney AL, Long JM, Hengemihle J, Mouton PR, Ingram DK, Jucker M (1998) Hippocampal neuron and synaptophysinpositive bouton number in aging C57BL/6 mice. Neurobiol Aging 19:599-606.

Chechik G, Globerson A, Anderson M, Young E, Nelken I, Tishby N (2002) Group redundancy measures reveal redundancy reduction in the auditory pathway. In: Advances in neural information processing systems 14 (Dietterich T, Becker S, Ghahramani Z, eds), pp 173-180. Cambridge, MA: Massachusetts Institute of Technology.

Dragoi G, Tonegawa S (2011) Preplay of future place cell sequences by hippocampal cellular assemblies. Nature 469:397-401.

Ekstrom AD, Meltzer J, McNaughton BL, Barnes CA (2001) NMDA receptor antagonism blocks experience-dependent expansion of hippocampal "place fields." Neuron 31:631-638.

Ekstrom AD, Kahana MJ, Caplan JB, Fields TA, Isham EA, Newman EL, Fried I (2003) Cellular networks underlying human spatial navigation. Nature 425:184-188.

Ferbinteanu J, Shapiro ML (2003) Prospective and retrospective memory coding in the hippocampus. Neuron 40:1227-1239.

Foldiak P (1992) The "ideal homunculus": statistical inference from neural population responses. In: Computation and neural systems (Eeckman FH, Bower J, eds), pp 55-60. Norwell, MA: Kluwer Academic Publishers.

Fox SE, Ranck JB Jr (1981) Electrophysiological characteristics of hippocampal complex-spike cells and theta cells. Exp Brain Res 41:399-410.

Frank LM, Brown EN, Wilson M (2000) Trajectory encoding in the hippocampus and entorhinal cortex. Neuron 27:169-178.

Frey MC, Sprengel R, Nevian T (2009) Activity pattern-dependent longterm potentiation in neocortex and hippocampus of GluA1 (GluR-A) subunit-deficient mice. J Neurosci 29:5587-5596.

Fuchs EC, Zivkovic AR, Cunningham MO, Middleton S, Lebeau FE, Bannerman DM, Rozov A, Whittington MA, Traub RD, Rawlins JN, Monyer H (2007) Recruitment of parvalbumin-positive interneurons determines hippocampal function and associated behavior. Neuron 53:591-604.

Fukaya M, Kato A, Lovett C, Tonegawa S, Watanabe M (2003) Retention of NMDA receptor NR2 subunits in the lumen of endoplasmic reticulum in targeted NR1 knockout mice. Proc Natl Acad Sci U S A 100:4855-4860.

Fyhn M, Molden S, Witter MP, Moser EI, Moser MB (2004) Spatial representation in the entorhinal cortex. Science 305:1258-1264.

Georgopoulos AP, Schwartz AB, Kettner RE (1986) Neuronal population coding of movement direction. Science 233:1416-1419.

Gothard KM, Skaggs WE, McNaughton BL (1996) Dynamics of mismatch correction in the hippocampal ensemble code for space: interaction between path integration and environmental cues. J Neurosci 16:8027-8040.

Hafting T, Fyhn M, Molden S, Moser MB, Moser EI (2005) Microstructure of a spatial map in the entorhinal cortex. Nature 436:801-806.

Hoffman DA, Sprengel R, Sakmann B (2002) Molecular dissection of hippocampal theta-burst pairing potentiation. Proc Natl Acad Sci U S A 99:7740-7745.

Hollup SA, Molden S, Donnett JG, Moser MB, Moser EI (2001) Accumulation of hippocampal place fields at the goal location in an annular watermaze task. J Neurosci 21:1635-1644. 
Jensen V, Kaiser KM, Borchardt T, Adelmann G, Rozov A, Burnashev N, Brix C, Frotscher M, Andersen P, Hvalby Ø, Sakmann B, Seeburg PH, Sprengel $\mathrm{R}$ (2003) A juvenile form of postsynaptic hippocampal long-term potentiation in mice deficient for the AMPA receptor subunit GluR-A. J Physiol 553:843-856.

Jezek K, Henriksen EJ, Treves A, Moser EI, Moser MB (2011) Thetapaced flickering between place-cell maps in the hippocampus. Nature 478:246-249.

Kennedy PJ, Shapiro ML (2009) Motivational states activate distinct hippocampal representations to guide goal-directed behaviors. Proc Natl Acad Sci U S A 106:10805-10810.

Kentros C, Hargreaves E, Hawkins RD, Kandel ER, Shapiro M, Muller RV (1998) Abolition of long-term stability of new hippocampal place cell maps by NMDA receptor blockade. Science 280:2121-2126.

Kjelstrup KB, Solstad T, Brun VH, Hafting T, Leutgeb S, Witter MP, Moser EI, Moser MB (2008) Finite scale of spatial representation in the hippocampus. Science 321:140-143.

Korotkova T, Fuchs EC, Ponomarenko A, von Engelhardt J, Monyer H (2010) NMDA receptor ablation on parvalbumin-positive interneurons impairs hippocampal synchrony, spatial representations, and working memory. Neuron 68:557-569.

Langston RF, Ainge JA, Couey JJ, Canto CB, Bjerknes TL, Witter MP, Moser EI, Moser MB (2010) Development of the spatial representation system in the rat. Science 328:1576-1580.

Lee I, Rao G, Knierim JJ (2004) A double dissociation between hippocampal subfields: differential time course of $\mathrm{CA} 3$ and $\mathrm{CA} 1$ place cells for processing changed environments. Neuron 42:803-815.

Leutgeb S, Leutgeb JK, Moser MB, Moser EI (2005) Place cells, spatial maps and the population code for memory. Curr Opin Neurobiol 15:738-746.

Mack V, Burnashev N, Kaiser KM, Rozov A, Jensen V, Hvalby O, Seeburg PH, Sakmann B, Sprengel R (2001) Conditional restoration of hippocampal synaptic potentiation in GluR-A-deficient mice. Science 292:2501-2504.

Markus EJ, Barnes CA, McNaughton BL, Gladden VL, Skaggs WE (1994) Spatial information content and reliability of hippocampal CA1 neurons: effects of visual input. Hippocampus 4:410-421.

McHugh TJ, Blum KI, Tsien JZ, Tonegawa S, Wilson MA (1996) Impaired hippocampal representation of space in CA1-specific NMDAR1 knockout mice. Cell 87:1339-1349.

McNaughton BL, Barnes CA, O’Keefe J (1983) The contributions of position, direction, and velocity to single unit activity in the hippocampus of freely-moving rats. Exp Brain Res 52:41-49.

Mehta MR (2004) Cooperative LTP can map memory sequences on dendritic branches. Trends Neurosci 27:69-72.

Mehta MR, Barnes CA, McNaughton BL (1997) Experience-dependent, asymmetric expansion of hippocampal place fields. Proc Natl Acad Sci U S A 94:8918-8921.

Mehta MR, Quirk MC, Wilson MA (2000) Experience-dependent asymmetric shape of hippocampal receptive fields. Neuron 25:707-715.

Morris RG, Garrud P, Rawlins JN, O’Keefe J (1982) Place navigation impaired in rats with hippocampal lesions. Nature 297:681-683.

Muir GM, Bilkey DK (2003) Theta- and movement velocity-related firing of hippocampal neurons is disrupted by lesions centered on the perirhinal cortex. Hippocampus 13:93-108.

Muller RU, Kubie JL (1989) The firing of hippocampal place cells predicts the future position of freely moving rats. J Neurosci 9:4101-4110.

Muller RU, Kubie JL, Ranck JB Jr (1987) Spatial firing patterns of hippocampal complex-spike cells in a fixed environment. J Neurosci 7:1935-1950.

Nakashiba T, Young JZ, McHugh TJ, Buhl DL, Tonegawa S (2008) Transgenic inhibition of synaptic transmission reveals role of CA3 output in hippocampal learning. Science 319:1260-1264.

O'Keefe J (1976) Place units in the hippocampus of the freely moving rat. Exp Neurol 51:78-109.

O'Keefe J, Conway DH (1978) Hippocampal place units in the freely moving rat: why they fire where they fire. Exp Brain Res 31:573-590.

O'Keefe J, Dostrovsky J (1971) The hippocampus as a spatial map. Preliminary evidence from unit activity in the freely-moving rat. Brain Res 34:171-175.

O'Keefe J, Nadel L (1978) The hippocampus as a cognitive map. Oxford: Clarendon.
O'Keefe J, Recce ML (1993) Phase relationship between hippocampal place units and the EEG theta rhythm. Hippocampus 3:317-330.

Olypher AV, Lánsk ý P, Muller RU, Fenton AA (2003) Quantifying location-specific information in the discharge of rat hippocampal place cells. J Neurosci Methods 127:123-135.

Pastalkova E, Itskov V, Amarasingham A, Buzsáki G (2008) Internally generated cell assembly sequences in the rat hippocampus. Science 321:1322-1327.

Pellegrini-Giampietro DE, Bennett MV, Zukin RS (1991) Differential expression of three glutamate receptor genes in developing rat brain: An in situ hybridization study. Proc Natl Acad Sci U S A 88: $4157-4161$.

Pouget A, Dayan P, Zemel R (2000) Information processing with population codes. Nat Rev Neurosci 1:125-132.

Rácz A, Ponomarenko AA, Fuchs EC, Monyer H (2009) Augmented hippocampal ripple oscillations in mice with reduced fast excitation onto parvalbumin-positive cells. J Neurosci 29:2563-2568.

Ranck JB Jr (1973) Studies on single neurons in dorsal hippocampal formation and septum in unrestrained rats. I. Behavioral correlates and firing repertoires. Exp Neurol 41:461-531.

Reisel D, Bannerman DM, Schmitt WB, Deacon RM, Flint J, Borchardt T, Seeburg PH, Rawlins JN (2002) Spatial memory dissociations in mice lacking GluR1. Nat Neurosci 5:868-873.

Rolls ET, Robertson RG, Georges-François P (1997) Spatial view cells in the primate hippocampus. Eur J Neurosci 9:1789-1794.

Romberg C, Raffel J, Martin L, Sprengel R, Seeburg PH, Rawlins JNP, Bannerman DM, Paulsen O (2009) Induction and expression of GluAl (GluR-A)-independent LTP in the hippocampus. Eur J Neurosci 29:1141-1152.

Rondi-Reig L, Petit GH, Tobin C, Tonegawa S, Mariani J, Berthoz A (2006) Impaired sequential egocentric and allocentric memories in forebrainspecific-NMDA receptor knock-out mice during a new task dissociating strategies of navigation. J Neurosci 26:4071-4081.

Rotenberg A, Mayford M, Hawkins RD, Kandel ER, Muller RU (1996) Mice expressing activated CaMKII lack low frequency LTP and do not form stable place cells in the CA1 region of the hippocampus. Cell 87:1351-1361.

Rutishauser U, Mamelak AN, Schuman EM (2006) Single-trial learning of novel stimuli by individual neurons of the human hippocampusamygdala complex. Neuron 49:805-813.

Sanderson DJ, Good MA, Skelton K, Sprengel R, Seeburg PH, Rawlins JNP, Bannerman DM (2009) Enhanced long-term and impaired short-term spatial memory in GluAl AMPA receptor subunit knockout mice: evidence for a dual-process memory model. Learn Mem 16:379-386.

Schmitt WB, Deacon RM, Seeburg PH, Rawlins JN, Bannerman DM (2003) A within-subjects, within-task demonstration of intact spatial reference memory and impaired spatial working memory in glutamate receptor-Adeficient mice. J Neurosci 23:3953-3959.

Schmitt WB, Sprengel R, Mack V, Draft RW, Seeburg PH, Deacon RM, Rawlins JN, Bannerman DM (2005) Restoration of spatial working memory by genetic rescue of GluR-A-deficient mice. Nat Neurosci $8: 270-272$.

Schmitzer-Torbert N, Jackson J, Henze D, Harris K, Redish AD (2005) Quantitative measures of cluster quality for use in extracellular recordings. Neuroscience 131: 1-11.

Siegel JJ, Nitz D, Bingman VP (2005) Spatial-specificity of single-units in the hippocampal formation of freely moving homing pigeons. Hippocampus 15:26-40.

Stanley GB, Li FF, Dan Y (1999) Reconstruction of natural scenes from ensemble responses in the lateral geniculate nucleus. J Neurosci 19:8036-8042

Tsien JZ, Huerta PT, Tonegawa S (1996) The essential role of hippocampal CA1 NMDA receptor-dependent synaptic plasticity in spatial memory. Cell 87:1327-1338.

Ulanovsky N, Moss CF (2007) Hippocampal cellular and network activity in freely moving echolocating bats. Nat Neurosci 10:224-233.

Wiener SI, Paul CA, Eichenbaum H (1989) Spatial and behavioral correlates of hippocampal neuronal activity. J Neurosci 9:2737-2763.

Wills TJ, Lever C, Cacucci F, Burgess N, O’Keefe J (2005) Attractor dynamics in 
the hippocampal representation of the local environment. Science 308:873-876.

Wills TJ, Cacucci F, Burgess N, O'Keefe J (2010) Development of the hippocampal cognitive map in preweanling rats. Science 328:1573-1576.

Wilson MA, McNaughton BL (1993) Dynamics of the hippocampal ensemble code for space. Science 261:1055-1058.

Wiltgen BJ, Royle GA, Gray EE, Abdipranoto A, Thangthaeng N, Jacobs N, Saab F, Tonegawa S, Heinemann SF, O'Dell TJ, Fanselow MS, Vissel B (2010) A role for calcium-permeable AMPA receptors in synaptic plasticity and learning. PloS One 5:pii:e12818.

Wood ER, Dudchenko PA, Robitsek RJ, Eichenbaum H (2000) Hippocampal neurons encode information about different types of memory episodes occurring in the same location. Neuron 27:623-633.
Yartsev MM, Witter MP, Ulanovsky N (2011) Grid cells without theta oscillations in the entorhinal cortex of bats. Nature 479:103-107.

Yu X, Yoganarasimha D, Knierim JJ (2006) Backward shift of head direction tuning curves of the anterior thalamus: comparison with CAl place fields. Neuron 52:717-729.

Zamanillo D, Sprengel R, Hvalby O, Jensen V, Burnashev N, Rozov A, Kaiser KM, Köster HJ, Borchardt T, Worley P, Lübke J, Frotscher M, Kelly PH, Sommer B, Andersen P, Seeburg PH, Sakmann B (1999) Importance of AMPA receptors for hippocampal synaptic plasticity but not for spatial learning. Science 284:1805-1811.

Zhang K, Ginzburg I, McNaughton BL, Sejnowski TJ (1998) Interpreting neuronal population activity by reconstruction: unified framework with application to hippocampal place cells. J Neurophysiol 79:1017-1044. 